\title{
Procedure for Application-Oriented Optimisation of Marine Propellers
}

\author{
Florian Vesting ${ }^{1, *}$, Rickard E. Bensow ${ }^{1}$, Rikard Johansson ${ }^{2}$, Robert Gustafsson ${ }^{2}$ \\ and Nicole Costa ${ }^{1}$ \\ 1 Department of Shipping and Marine Technology, Chalmers University of Technology, \\ 41296 Gothenburg, Sweden; rickard.bensow@chalmers.se (R.E.B.); nicole.costa@chalmers.se (N.C.) \\ 2 Rolls-Royce Hydrodynamic Research Centre, Rolls-Royce AB, PO Box 1010, 68129 Kristinehamn, Sweden; \\ rikard.johansson@rolls-royce.com (R.J.); robert.gustafsson@rolls-royce.com (R.G.) \\ * Correspondence: florian.vesting@chalmers.se; Tel.: +46-31-772-2694
}

Academic Editor: Moustafa Abdel-Maksoud

Received: 9 June 2016; Accepted: 28 October 2016; Published: 26 November 2016

\begin{abstract}
The use of automated optimisation in engineering applications is emerging. In particular, nature inspired algorithms are frequently used because of their variability and robust application in constraints and multi-objective optimisation problems. The purpose of this paper is the comparison of four different algorithms and several optimisation strategies on a set of seven test propellers in realistic industrial design setting. The propellers are picked from real commercial projects and the manual final designs were delivered to customers. The different approaches are evaluated and final results of the automated optimisation toolbox are compared with designs generated in a manual design process. We identify a two-stage optimisation for marine propellers, where the geometry is first modified by parametrised geometry distribution curves to gather knowledge of the test case. Here we vary the optimisation strategy in terms of applied algorithms, constraints and objectives. A second supporting optimisation aims to improve the design by locally changing the geometry, based on the results of the first optimisation. The optimisation algorithms and strategies yield propeller designs that are comparable to the manually designed propeller blade geometries, thus being suitable as robust and advanced design support tools. The supporting optimisation, with local modification of the blade geometry and the proposed cavity shape constraints, features particular good performance in modifying cavitation on the blade and is, with the AS NSGA-II (adaptive surrogate-assisted NSGA-II), superior in lead time.
\end{abstract}

Keywords: marine propeller; multi-objective optimisation; cavitation constraints; evolutionary algorithm; PSO

\section{Introduction-The Problem in Propeller Design}

Suppliers in the competitive market of ship propellers and propulsion have experienced a change in customers' awareness for the selection criteria of an order. For merchant vessels it has predominantly been a question of investment cost to choose which supplier may deliver the propeller. The situation shifted during the last couple of years towards a situation where also the efficiency and fuel consumption became major selection criteria for the customer. With the expansion of routing in ecological sensitive areas, a recent concern is the propeller radiated noise level. However, in general, concern for operational and maintenance costs, environmental awareness and emission regulations have sharpened the perception in the merchant fleet, such that suppliers are more often forced into comparative performance races [1].

Competition between contestants is generally good since it ensures the customer to receive the product that fulfils the requirements best; and to choose from products that are obtained by different strategies and design philosophies of the competitors. However, in recent competitive situations, 
the designated winner is often the supplier that delivers the highest efficiency and thereby the lowest fuel consumption. The verification is commonly done by an impartial test facility, at model scale with self-propulsion tests and converted to full-scale according to standard procedures, e.g., the ITTC procedure. This competition and evaluation practice initiated a trend of sub-optimising the propeller designs towards efficiency performance and disregarding other performance characteristics [1]. Such practice yield designs that are unsatisfying in operation and may ultimately end in failure of the equipment, e.g., due to cavitation erosion.

Propellers are highly adapted to the flow around the specific ship it is designed for and its operation conditions, to achieve high levels of efficiency. However, to avoid sub-optimal propellers that achieve the lowest power consumption yet ignores other performance characteristics, the designer has to consider requirements which are not only contradictory to efficiency but also have practical limitations. Since the propeller experiences varying inflow conditions while travelling through the circumferential wake, cavitation and propeller induced pressure pulses are the most evident propeller effects, contradictory to efficiency. To satisfy the shipowners' expectations and to deliver a practical design, the designer has to consider and control the cavitation, and constrain static and dynamic blade stresses, classification requirements and in case of controllable pitch propellers, hub strength and blade clearance.

Thus, propeller design is truly an art of compromising the performance and requirements in an iterative manner, with the final propeller geometry being a unique design. This is naturally a multi-objective optimisation task with constraints, intimately connected with multi-disciplinary numerical evaluation methods (e.g., for cavitation or stresses). The various requirements lead to a large number of alternatives to be studied and thereby put restrictions on the numerical analysis tools. The challenging factor for the designer is to develop a propeller that fulfils the requirements and expectations within a short time frame available in the competition races, before manufacturing of the supplier's model propeller candidate. Thus, although the evaluation of propeller performance demands highly unsteady and physically tremendously complex simulations, it is common practice to apply less accurate but faster potential methods (Mishima [2], Griffin and Kinnas [3], Lee et al. [4], Bertetta et al. [5]).

Automated propeller optimisation emerges more frequently to produce a large number of design alternatives and thereby support the designer during the design synthesis. Foeth [6] presents propeller geometry parametrisation based on database records and the multi-objective optimisation in behind condition, which applies the derived parameters. Thus, the optimisation is carried out with full interaction between the propeller changes and flow around the hull. However, this is computationally intensive and no constraints are considered. It is hypothesised that a preliminary optimisation is carried out to determine the main parameter ranges. Berger et al. [7] present such idea in a two-stage optimisation with propeller hull interaction applied in the second stage, after evaluation of a multi-objective optimisation in given wake condition. This is an efficient intermediate step, also indicated by Foeth [6], to assist the designer in finding the optimal design for a given hull geometry. However, in [7], only a constraint on thrust equality is applied and the objective function is biased by the applied weight factor.

Constrained propeller optimisation is , e.g., presented by Vesting and Bensow [8], with a multi-objective propeller optimisation in behind condition, considering sheet cavitation, or by Han et al. [9] with a multi-objective propeller blade optimisation in given wake and sheet cavitation as a constraint. Similarly, Kamarlouei et al. [10] apply an optimisation in fixed wake, with cavitation constraints on the blade loading (Keller criterion) and the two-dimensional cavitation behaviour of one blade section by the use of the cavitation bucket diagram, from which the cavitation-free area can be identified. Kamarlouei et al. [10] also include, unlike Han et al. [9], constraints for the blade strength, though with simplification to cantilever beam theory. Bertetta et al. [5] present the multi-objective optimisation of a controllable pitch propeller (CPP), with the main aim to reduce the face cavitation and thereby the resultant radiated noise. Common denominator of the enumerated examples is the applied numerical evaluation of the propeller performance, which is based on potential methods. However, applied 
cavitation constraints are based on general characteristics, e.g., the maximum cavitation volume or the cavity areas, and constraints on blade strength are only included by Kamarlouei et al. [10].

This shows a clear lack of optimisations applying practical limitations and thereby an efficient constraint handling and integration in the optimisation algorithms. Puisa and Streckwall [11] present a constrained propeller optimisation with an adapted constraint-handling technique. Results are compared with the standard non-dominated sorting genetic algorithm (NSGA-II) by Deb et al. [12], which show that the problem adapted constraint handling is superior. The applied constraints on cavitation are, however, conservative and based on the circumferential mean wake, and prohibit cavitation occurrence at all. Hence, they are not entirely practical for a broad range of propellers.

We have developed and applied new constraints for sheet cavitation, based on potential flow analysis and two novel optimisation algorithms based on the NSGA-II and the standard particle swarm optimisation (PSO) algorithm [13]. We proposed, in addition, a constraint handling method for infeasibility handling, which is adapted to manual propeller design. In this paper, we present their application to a broad range of test propellers and evaluate their case dependent performance, in order to develop guidelines for efficient propeller optimisation. All optimisations additionally include cavitation constraints, limitations on blade strength, class regulations and geometrical blade limitations. We also propose a recommended optimisation procedure for marine propellers.

We will first introduce the objectives for the propeller design task and outline the computational methods, before a typical manual design process is explained for the design analysis and optimisation phase. Subsequently, in Section 4 we elaborate the automated design procedure with a summary of the applied algorithms, constraints and constraints handling routine. In Section 5 we provide information about the test cases and the different optimisation strategies which is followed by the results discussion.

\section{Propeller Design Objective and Analysis Tools}

What is the optimal propeller design? To answer this question we have to interrogate the purpose of the propeller and ship it needs to be designed for. The optimal propeller performs certainly different for a container vessel than for an icebreaker, a naval, or a cruise vessel. Depending on the perspective, strength or reliability are the critical characteristics (e.g., icebreaker or naval vessels) while in other applications low noise level is the most important factor (e.g., cruise vessels). However, on a certain level all applications share the same interest for low fuel consumption, less vibrations due to propeller induced pressure pulses, safety margin for cavitation erosion and classification conformity. Therefore we restrict the variation of the optimisation set-ups to the same objective of minimisation of pressure pulses and maximisation of propeller efficiency. Both are common characteristics in propeller design which can be measured in model and full scale, and are therefore often contractually guaranteed. Additionally, we apply constraints on blade stress, cavitation, classification regulations and manufacturing limitations. These objectives and constraints are interconnected and often contradictory which requires a simultaneous consideration during the optimisation. If the propeller is optimised disregarding some characteristics the result will be sub-optimal, and an adjustment of the optimal solution will only be patchwork.

We outline a typical design procedure for the analysis and optimisation phase to find the optimal blade geometry for a given wake, inspired by Rolls-Royce design philosophy and evaluation tools. The wake is in this procedure corrected to full scale and to include the effect of a propeller. As the initial step, a lifting line method, with lifting surface correction, is employed to deliver the initial pitch and camber distribution, which is determined from the optimal circulation distribution, according to Lerbs induction factor method [14].

For the evaluation of the propeller performance, we focus on the use of moderately low fidelity numerical tools to be utilised during the early design phase. For the propeller performance, including the prediction of sheet cavitation, we utilised the vortex lattice method "MPUF-3A" [15]. This methods offer sufficient accuracy to compare the results in relation with each other and a benchmark, in a reasonable 
short evaluation time. The propeller blade geometry is represented by a lattice of discrete vortices distributed on the mean camber surface, which have to be determined from boundary conditions. First development of this lifting surface prediction method was presented by [16]. The method was extended towards an unsteady analysis tool for cavitating flow [17]. Further development, e.g., by introducing distinct set of source sheets for a blade thickness distribution and leading edge correction $([3,18,19])$, yield the current version of the tool, which also includes among other features, wake alignment, mid-chord cavitation, thickness-load coupling and hub influence.

The propeller induced pressure pulses are calculated in close relation to the MPUF-3A propeller performance computation. The applied tool is a boundary elements method to compute the pressure amplitudes of the first three blade harmonic frequencies. The method solves the diffraction potential on a flat hull-dummy surface, by applying Green's formula [20]. However, the objective is the maximal amplitude of the first blade frequency which occurs on one of the surface panels.

Today's propeller geometry feature commonly high skew and curved rake distributions and evolve in a highly complex three-dimensional shape. Thus numerical method for the static stress level and the dynamic strength analysis require finite element methods (FEMs). Here we apply a code which uses shell elements of quadrilateral shape, generated on the mid-surface of the blade. Evaluated are the maximum von Mises stresses, which are evaluated at a generic strength section above the blade root filling.

\section{Manual Propeller Design}

A common practice in propeller design is to develop a preliminary design concept based on best knowledge and a design database before signing the contract with the customer, and improve this design successively to find the best compromise between the competing objectives and constraints. Hence it is naturally a multi-objective optimisation task in which various constraints, intimately connected with numerical methods (e.g., for cavitation or stresses), need to be accepted and considered.

Figure 1 demonstrates what the regular manual propeller design process involves. Input variables mainly provided by the customer are allocated in the database, resulting in a number of preliminary design outputs (principally regarding shaft line) which may be saved or discarded. From the database, the propeller designer can select the desired outputs and import them into the existing software, where propeller geometry is tweaked in two separate geometry modules. This depends not only on the customers' requirements but also on the designers' knowledge and experience. Once the geometry is fixed, the designer runs the analysis modules followed by the strength analysis modules. After obtaining the design results, if they are not entirely satisfactory, the designer may go back to the first or second geometry modules and iterate by modifying geometric parameters, until a fitting result is found. Although not usual, the designer may even go back to the preliminary design and modify shaft line characteristics.

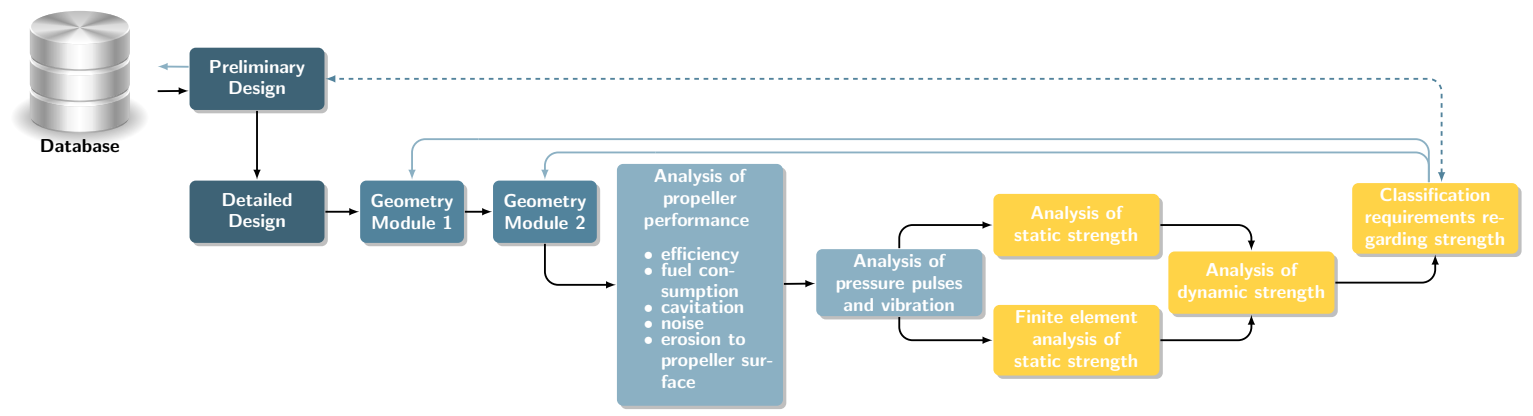

Figure 1. Manual design process and iteration.

The propeller geometry is during the outlined procedure developed towards, what is believed, the optimal design. Running the iterations and making decisions closely depends on the experience and skill of the designer, where time available for the design iteration is the limiting factor. Thus, the 
number of tested design alternatives and the lead time of analysis tools is limited. Commonly around 30 variants in 3 to 8 iterations are investigated, which can readily be reduced to less variants in case of urgency.

\section{Automated Propeller Design Procedure}

Automated propeller optimisation emerges more frequently. However, it is often very ambitious regarding computational effort in case entirely viscous CFD simulations are applied, as described by Foeth [6]. The ideal case for a propeller optimisation would include the optimisation of the hull geometry. A designer should iteratively account for interaction effects between the propeller and the hull. But this broadens the design space significantly and requires cooperation between the hull and the propeller designer as well as high fidelity simulation of the hull surrounding flow. Thus, we concentrate our optimisation on the most common situation in which a designer needs to find the best propeller design for a given hull form.

We adapted common optimisation algorithms (NSGA-II and PSO) to three algorithms for propeller design and implemented and applied six optimisation strategies [13] which are in this paper introduced to a broader range of propeller types. The algorithms are implemented in the design toolbox at Rolls-Royce Hydrodynamic Research Centre and thereby have access to all analysis tools that are part of the manual design process. Thus we have access to alternate objectives, geometrical limitation, e.g., for CPP, classification regulations and various analysis tools which differ in fidelity level.

Beside the standard NSGA-II, Deb et al. [12], we apply two modified versions of the NSGA-II, which are assisted by a meta-model (Kriging model [21]), implemented in the DACE MATLAB toolbox, Lophaven et al. [22]. The first algorithm, the Adaptive Surrogate-assisted NSGA-II (AS NSGA-II) evaluates variants on the meta-model and updates the meta-model frequently every fourth generation with calculated results. The second algorithm, the Surrogate-Assisted Neighbourhood Assessment NSGA-II (SANA NSGA-II) evaluates all individuals in the population with calculated results. Yet before a new generation is evaluated, the SANA NSGA-II assesses the neighbourhood of each individual with the help of the meta-model and selects the most promising design as the individual to calculate. The third algorithm applied is a Particle Swarm Optimisation (PSO) algorithm which is a combination of the NSGA-II and a standard PSO algorithm by Kennedy and Eberhart [23] algorithm. Hence, our PSO is capable of multiple objectives and non-dominated sorting and utilises an archive of best solutions which continuously updates the rank and crowding distance, with respect to the latest swarm. A detailed explanation of the algorithms is presented by Vesting et al. [13].

The optimisation results in different designs when different strategies are applied regarding the selection of parameters modification, objective and constraint selection, initialisation and handling of infeasibility. In general we apply constraints on the required thrust, the blade strength, classification regulations and geometry limitations. Additionally constraints on the cavity shape and their location are applied to (i) enhance objectives sensitivity to the cavitation on the blade, predominantly propeller induced pressure pulses; and (ii) to monitor the formation of the cavity shape and thereby reduce the risk for potentially erosive cavitation. In particular, the constraints target the cavity sheet thickness at the blade tip, the maximal cavitation volume as well as its length in chord-wise direction and the change in cavity volume between two consecutive time steps. We have additionally developed new constraints which rate the shape of the cavity and assign a harm-factor according to the similarity of the cavity closure line to a convex closure line and the location of the maximum sectional cavity centroid (e.g., harmful at the tip, root and downstream in chord-wise direction) [13]. We summarize the cavitation constraints with a brief description in Table 1. 
Table 1. Cavity shape constraints.

\begin{tabular}{ll}
\hline Constraint & Description \\
\hline CMaxVol & Maximum non-dimensional cavity volume at a single blade position. \\
\hline Tip 1,2,3 & Cavitation thickness at the three outermost blade radii . \\
\hline CCent harmfactor & $\begin{array}{l}\text { Calculates the chord-wise centroid from the finite set of cavity thickness at each } \\
\text { span-wise section and assign a harm-factor for the furthest downstream position } \\
\text { to rate that position. The blade is divided into harmless and harmful sectors and } \\
\text { the harmfactor is accumulated. }\end{array}$ \\
\hline Closure harmfactor & $\begin{array}{l}\text { Cavity closure line compares the normalised (length between 0 and 1) cavity } \\
\text { trailing edge with a given, faultily example for a closure line. Such example of } \\
\text { distinct convex shape is given by a normalised polynomial. The pairwise difference } \\
\text { between the given curve and the sections-wise cavity length are compared and } \\
\text { yield a root mean square (RMS) error. A small RSM imply therefore a high } \\
\text { similarity to a convex shape and vice verse. }\end{array}$ \\
\hline Cavity change & $\begin{array}{l}\text { Fluctuations of the cavity volume. Maximal change in non-dimensional volume } \\
\text { between two time steps. }\end{array}$ \\
\hline CLength & Maximal non-dimensional length of each cavitating blade section. \\
\hline
\end{tabular}

Unlike the standard optimisation algorithms, which commonly assign a constraint violation independent from the degree of violation, we utilise for each constraint a violation measure relative to the performance of the current population. Thus, a solution is assigned with a value of -1 if it features the worst constraint performance in the current population and 1, vice versa. The other individual are assigned relative to the best and worst performance respectively. The assessment is repeated with each constraint and eventually the constraint measure is accumulated in the Constraint Violation Measure (CVM) which determines the feasibility of a solution. A solution can thereby be feasible to the algorithm even if one or more constraints are marginal violated [13]. A designer judges a design differently on the level of violation and rates constraints unequal and is possibly interested in designs that perform excellent although they might be marginally infeasible, to find the next design step. The procedure we utilise, is intended to feature the designer's perspective in a manual design process.

The strategies we pursue are directional classified in a 2-stage optimisation proposal to efficiently accomplish the propeller design, Figure 2 . The complexity of the design procedure makes a systematic optimisation procedure valuable. We assume in this procedure that one starts from a preliminary design concept, based on project estimations. Thereby, we imply that the operation condition (e.g., RPM, ship speed etc.) and the main propeller dimensions, like the propeller diameter, are already determined; it is about to find the wake adapted blade geometry. A sectional shape variation is out of the scope of this paper to contain the design space.

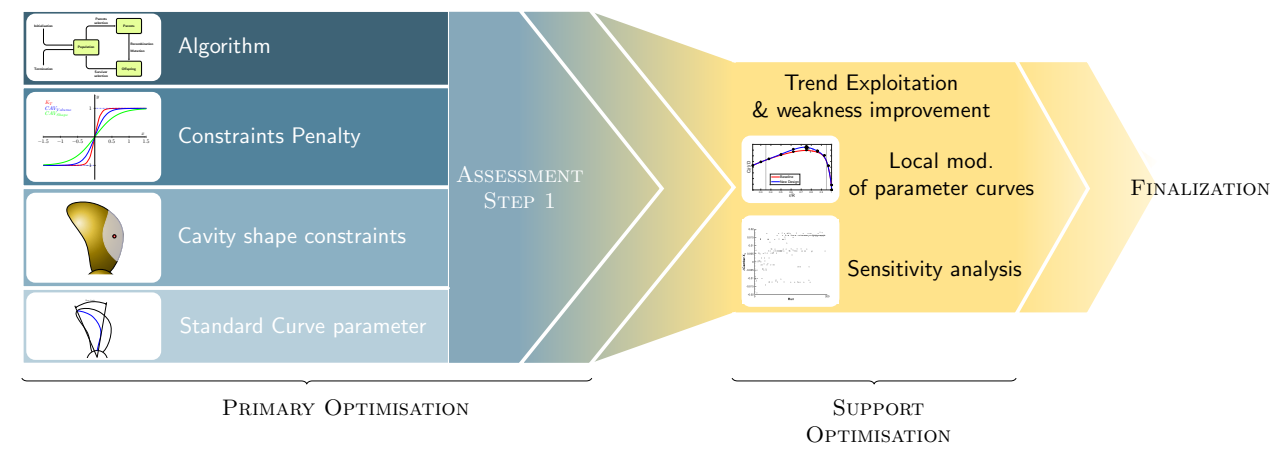

Figure 2. Two-stage propeller optimisation. 
In the first primary optimisation the propeller geometry is modified using parametric geometry distribution curves. These geometry distributions are related to the propeller and its application type and are controlled by general input parameters, e.g., blade area ratio, skew at the blade tip or rake at the tip. Such standard distribution curves are based on experience and design philosophy and provide the first draft of the design, similar as described by Foeth [6]. Here we utilize the Rolls-Royce standard distribution curve parameters which may be different for competitors, yet the optimisation strategies will still be the same. Table 2 provides an overview of the parameter distribution curves and their corresponding control parameters. The primary optimisation makes use of these global parameters to provide an insight into the propeller response and thereby uncover tendencies and parameter influences in a subsequent assessment step done by the designer.

Table 2. Standard distribution curves and their corresponding controlling parameters.

\begin{tabular}{|c|c|c|}
\hline Parameter Curve & Control Parameter & Description \\
\hline $\mathrm{L}$ & EAR & $\begin{array}{l}\text { Modification of EAR to generate } \\
\text { different chord Length distributions. }\end{array}$ \\
\hline SKEW & Tip & $\begin{array}{l}\text { Modification of SKEWLINE by } \\
\text { changing the skew at Tip. (degrees) }\end{array}$ \\
\hline SKEW & LFHub & $\begin{array}{l}\text { Modification of SKEWLINE by } \\
\text { changing the LF Hub value. (mm) }\end{array}$ \\
\hline TMAX & Tred07 & $\begin{array}{l}\text { Modification of TMAX curve by changing } \\
\text { the thickness reduction at } 0.7 \mathrm{R} .(\%)\end{array}$ \\
\hline RAKE & RkTip & $\begin{array}{l}\text { Modification of RAKE curve by changing } \\
\text { the rake at the tip }(\mathrm{mm}) \text {, positive towards } \\
\text { the propeller face side. }\end{array}$ \\
\hline $\mathrm{Pi} / \mathrm{D}$ & Unload factor root & $\begin{array}{l}\text { Modification of the load distribution at the root } \\
\text { by changing how unloaded it should be. } \\
(0.0=\text { No unloading, } 1.0=\text { Full unloading) }\end{array}$ \\
\hline $\mathrm{Pi} / \mathrm{D}$ & Unload factor tip & $\begin{array}{l}\text { Modification of the load distribution at the tip } \\
\text { by changing how unloaded it should be. } \\
(0.0=\text { No unloading, } 1.0=\text { Full unloading) }\end{array}$ \\
\hline $\mathrm{Pi} / \mathrm{D}$ & Unload width factor & Influence zone for adjustment of load distribution. \\
\hline
\end{tabular}

The designer selects also a suitable optimisation algorithm, the objectives, constraints and whether constraints are handled with amplified penalties or not. The combination of constraints is crucial for the CVM and requires the designer's experience to rate the achievable performance compared to the baseline design. In this paper we go beyond the scope of a primary optimisation by applying several strategies (as outlined in Section 5) in the primary optimisation to develop guidelines for their application. The intention for a practical application is, however, to utilise only one of the strategies in the primary optimisation. The selection of which strategy to use depends consequently on the performance of the baseline, e.g., cavitation extent or constraints which are challenging. The groups in Table 4 introduce a categorisation of the strategies: Group 1 differs in application of optimisation algorithms, Group 2 differs in constraints setting and Group 3 essentially in geometry modification method. Groups 1 and 2 correspond to a primary optimisation and Group 3 to support optimisation. The settings in Table 4 are elaborated in Section 5.

Support optimisation is applied to use the gathered knowledge on trends and parameter influences for the detailed design. Parameter curves with high impact on constraints or objectives are used for local blade geometry modification to further explore and exploit obtained trends or to improve the performance of constraints, e.g., together with cavity shape constraints locally manipulate the cavity. Local modifications are applied indirectly to the B-spline parameter distribution curves by modifying two 4th order Bézier curves which supply an offset to the B-spline curve control points. The desired parameter value is iteratively achieved until the parameter curve passes the desired point. A width 
factor determines in the process the span wise perturbation of the parameter curve and applies thereby smooth modifications to the original curve [13].

\section{Test Cases}

The Rolls-Royce Hydrodynamic Research Centre (RRHRC), Kristinehamn, provided in total seven propeller designs for this study. Table 3 provides an overview of the propeller outline, their design purpose and main characteristics. The designs represent common applications and a wide range of geometry, e.g., blade area (EAR 0.4-0.7), diameter (D 3-7 m), skew $\left(6^{\circ}-45^{\circ}\right)$ and design pitch (P/D 1.05-1.4). Apart from Opt-6 all propellers are delivered designs and already passed through a manual optimisation. Thus for the optimisation assignment we "reset" each design to an early project calculation status, considering however, final settings like hub specification and possibly changed operation conditions according to the delivered design. Hence we have for each propeller one benchmark design (the delivered design) and an initial design (the baseline) to compare the final results of the optimisations with. Elaboration on design details and absolute values will be omitted in this paper for protection of commercial interests of Rolls-Royce AB.

Table 3. Benchmark propeller and their characteristics.

\begin{tabular}{|c|c|c|}
\hline Propeller & Characteristics & Category \\
\hline 1 & $\begin{array}{l}\text { - twin-screw cruise vessel } \\
\text { - strict requirements on high } \eta \text { and low } p \text { with contract penalty } \\
\text { - risk for tip vortex inception } \\
\text { - CPP }\end{array}$ & Performance \\
\hline 2 & $\begin{array}{l}\text { - FPP propeller for container vessel } \\
\text { - integrate rudder and propeller (hub cap and propulsion bulb) } \\
\text { to optimise hydrodynamic efficiency } \\
\text { - high requirements on } \eta\end{array}$ & Performance \\
\hline 3 & $\begin{array}{l}\text { - naval vessel } \\
\text { - highly loaded } \\
\text { - limitation on draft and propeller-hull clearance } \\
\text { - high risk for erosive mid-chord cavitation }\end{array}$ & Constraints \\
\hline 4 & $\begin{array}{l}\text { - } \text { cruise vessel } \\
\text { - Mermaid pod, i.e., electric rotatable thruster } \\
\text { - high requirements on } \eta \\
\text { - low stresses at normal straight inflow }\end{array}$ & Performance \\
\hline 5 & $\begin{array}{l}\text { - } \text { Ro-Pax vessel } \\
\text { - high ice class requirement (LRS 1A Super) } \\
\text { - high demand on low } p \\
\text { - risk for mid-chord and root cavitation }\end{array}$ & Constraints \\
\hline 6 & $\begin{array}{l}\text { - naval vessel } \\
\text { - highly loaded } \\
\text { - design alternative of Opt-3 } \\
\text { - risk for travelling cavities } \\
\text { (convex closure line of the sheet cavitation) }\end{array}$ & Constraints \\
\hline 7 & $\begin{array}{l}\text { - } \text { cruise vessel } \\
\text { - upgrade to an existing vessel to reduce fuel consumption } \\
\text { - } \text { high requirements on } \eta \\
\text { - } \text { risk for high blade stresses } \\
\text { - no cavitation simulation }\end{array}$ & Constraints \\
\hline
\end{tabular}


The optimisation cases are divided into three groups in which the first two groups emphasise on the primary optimisation according to Figure 2. Here we investigate general qualities of optimisation algorithms by altering the optimisation algorithms and utilise the parametrised geometry curve for variation, which are introduced in Table 2 . Table 4 provides an overview of the different settings for several optimisation cases. Each column (Opt-X-1 to Opt-X-15 where $\mathrm{X}$ represents each propeller respectively) represents one strategy set-up from which the majority is applied to each of the test propellers. The selection of set-ups to apply in Group 2 and 3 depends on the outcome of Group 1 optimisations. Hence, optimisations in Group 2 and Group 3 are conducted subsequent to Group 1.

Table 4. Optimisation test matrix with alternating algorithms, geometry variation and constraint settings. (C: Applied in case of cavitation optimisation; E: Applied in case of efficiency performance optimisation.)

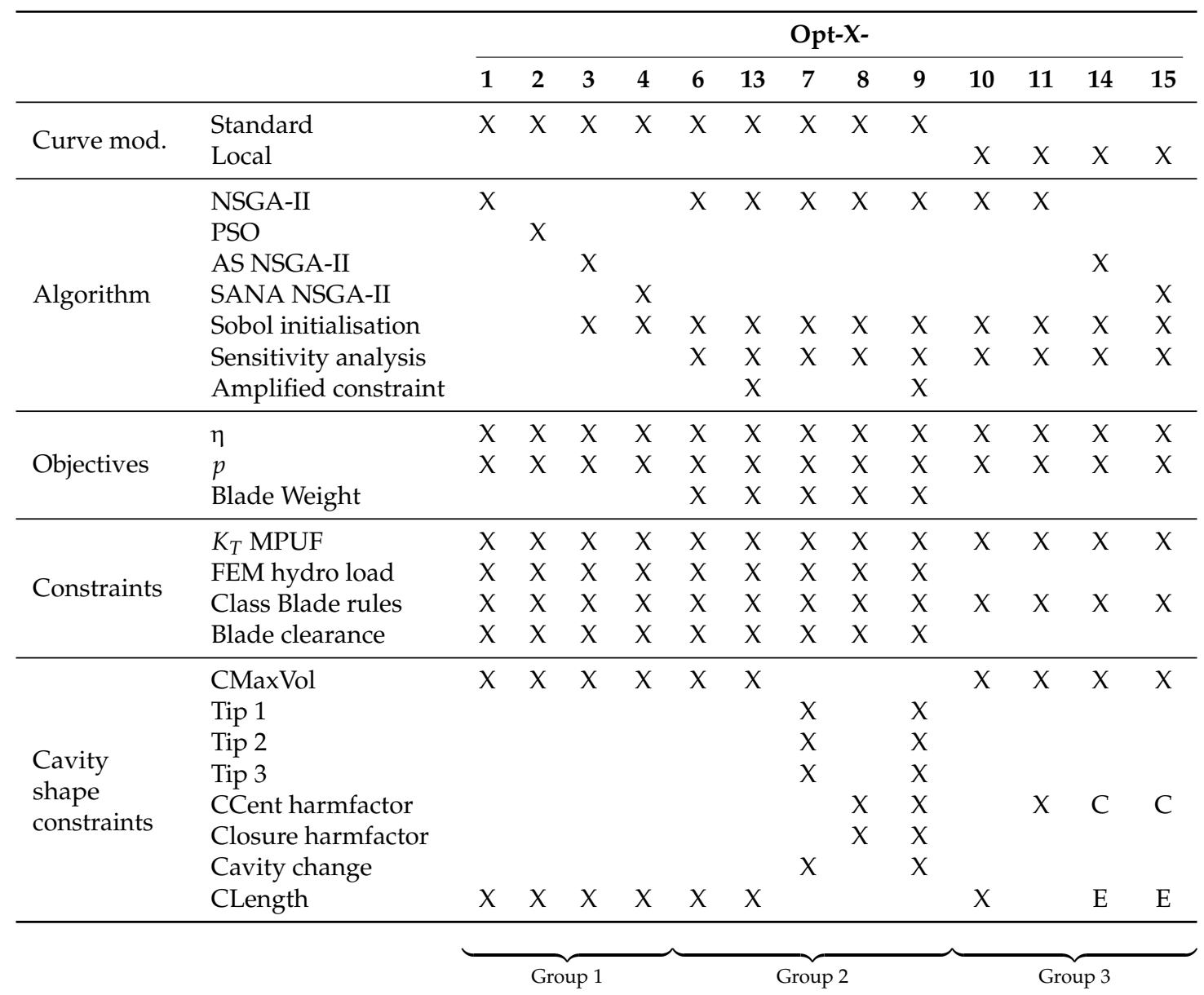

For optimisations in Group 2 (Opt-X-6 to Opt-X-9), we reduce the number of input parameters by observations of Group 1 and the results of a sensitivity analysis on the parameter influence. Opt-X-6 provides the basis with fewest modification compared to Group 1. For Opt-X-7, Opt-X-8 and Opt-X-9 we, additionally, introduce cavity shape constraints which are alternated among the cases. Optimisations Opt-X-13 and Opt-X-9 are conducted subsequent and utilise amplification on constraints which are frequently violated in earlier cases. Group 2 still belongs to the primary optimisation, yet it is composed of different optimisation strategies which carefully have to be selected by the designer, in accordance to the observations on the baseline design.

The principle difference for Group 3 is the application of local modification of blade parameters and the aim to particular improve either objectives or cavity shape. Hence, in Group 3 we apply either Opt-X-10 for objective improvement or Opt-X-11 for cavitation improvement. Opt-X-14 and Opt-X-15 are conducted supplementary according to either Opt-X-10 or Opt-X-11 to judge the capability of meta-model assisted optimisations for the local blade modifications. 
For all optimisation cases we specify the same settings for the optimisation algorithms with settings for population size $\left(N_{I}=24\right)$, number of generations $\left(N_{G}=40\right)$, mutation probability $\left(p_{m u t}=0.75\right)$, crossover probability $\left(p_{\text {cross }}=0.15\right)$ and, for the PSO, inertia weight $(w=0.4)$. Solely the population size and the number of generations is revised in case of Group 2 and 3 where the number of parameters is reduced. Parameter limits are generally set to a maximal variation of $\pm 10 \%$ to the initial value of the baseline design.

However, special consideration is given to parameters like EAR for cases Opt 3 and Opt 5 . The initial designs provide already a high blade area ratio. Hence, an increased value up to $+10 \%$ yields, as expected, blade collision and other hub related problems. Thus, the upper limit is revised to meet the geometric requirement. The same holds for the rake parameter since this value is given in $\mathrm{mm}$ which results in too few variation within the $10 \%$ range. Consequently, we apply a wider range of $1000 \%$ compared to the baseline. The constraint limits are similar related to the baseline design with a limit of $5 \%$ improvement on cavitation and blade stress constraints. Only Opt-7 requires special consideration of the blade stress constraint since the baseline yields unacceptable high stresses and consequently the limit is set according to the benchmark design. The required thrust constraint $\left(K_{T}\right)$ is as well set according to the benchmark design, to ensure a fair comparison with the benchmark design.

\section{Results and Discussion}

The optimisation survey involves the results of up to 15 optimisation set-ups for each of the seven propellers, which adds up to more than 80 optimisations and thereby a comprehensive number of potential optimal solutions that require assessment. Consequently, there is a need for qualitative and quantitative criteria to determine the convergence and quality of the optimisation itself, before appraising the optimal solution. In general we classify the assessment into three assessment ranks:

- Potential of the optimal solutions, to rate if the optimisation was actually able to improve the design.

- Convergence of the selected set-up, to rate the confidence of the optimisation to actually end up in a specific location, the global optimum, and thereby provide information about the computation effort of the optimisation approach.

- Constraints feasibility, to judge if the approached optimal solutions comply with the constraints.

We divide the result presentation further according to the categories in Table 3 for either performance or constraint optimisation. Each propeller will be assessed separately, starting with a summary table of observations according to the assessment ranks of potential improvement and convergence followed by a discussion of constraints feasibility and comparison with the benchmark design. To examine the central tendency of input and output quantities, we evaluate predominantly the medians within the population of one generation. The median is a more robust measure of the location parameter in case of skewed or non-normal distributions. It reflects the central development of a parameter or objective over the progress of the optimisation remarkably well since it reduces the importance to outliers.

\subsection{Performance Optimisation}

The propeller Opt-1, Opt-2 and Opt-4 require thoughtfulness regarding the objectives due to e.g., contract penalties. These propeller are optimised to enhance the objective performance ( $\eta$ and $p$ ). In Groups 2 and 3 we select parameters with expected importance for the objectives (e.g., pitch and chord) and according to the parameter sensitivity.

\subsubsection{Opt-1}

Common geometry developments, among all optimisations on Opt-1, are exclusively obtained for the rake, which increased, and EAR, which decrease. Opt-1-3 decreases the blade area with $10 \%$ down to the parameter limitation, which is misled by an optimistic estimation of pressure pulse, cavitation and blade strength constraints (Figure A1a and Figure 3a). Both blade stresses and cavitation are up 
to $10 \%$ better estimated by the meta-model than simulated. Opt-1-7 and Opt-1-8 decreases its EAR parameter to a similar low level and thereby improved the blade weight objective in Group 2. Both fail, however, as a consequence in adherence of the blade strength constraint (Figure 3b).

Constraints development is given by the CVM in Figure 4 and the median of the constraint values in Figure 3. These figures reveal that the results of Opt-1-1 and Opt-1-6, with fair improvements according to objectives medians (Figures A1 and A2) are virtually infeasible after generation 20, although all constraints are feasible. The only constraint that gently declines from $5 \%$ to $4 \%$ after 10 generations is $K_{T}$ for Opt-1-1 (Figure 3a). A detailed analysis of the constraints of Opt-1-6 show that this is due to a steep increase in blade stress within the first 10 generation up to $10 \%$ higher than the baseline design (Figure 3b).

The algorithm tries to counteract the decline which leads to a sudden increase of cavitation volume by $5 \%$ at generation 13 and high blade stresses. However, this should still result in a positive CVM but is prevented by an increasing number of failed simulations, with the consequence of unreliably small values of $K_{T}$ and annulled cavitation. Since the CVM is a relative measure, depending on each the maximum and minimum constraint values within a generation, the consideration of such outliers yield small or negative violation measures for the other solutions in that generation. Our CVM has thereby the additional effect of penalising failed simulations.

To compare the influence of different cavitation constraints, we have to select particular designs from the Pareto optimal solutions for each case in Group 2. This is done manually to find the best trade-off among the objectives and to ensure that the $K_{T}$ constraint is to the greatest possible extent fulfilled, i.e., we except a higher $K_{T}$ of up to $4 \%$ compared to the required $K_{T}$ for equilibrium. Both selected solutions of Opt-1-6 and Opt-1-7 violate, however, the blade strength constraints with $9.2 \%$ and $3.4 \%$, as indicated by the median assessment (Figure 3). The advantage of Opt-1-7 is otherwise a significant cavitation volume reduction of about $20 \%$. From Figure 5 it is visible that this case is the only result in Group 2, where the cavitation thickness at the blade tip is reduced. Opt-1-7 utilises cavitation constraints particularly suited for the sheet thickness at the three outermost blade radii $(r / R \geq 0.95)$. Both Opt-1-6 and Opt-1-7 introduce geometrical changes that yield mid-chord cavitation (Figure $5 a, b)$. This cavitation is on the other hand prevented in case of Opt-1-8 using the centroid harm-factor constraint.
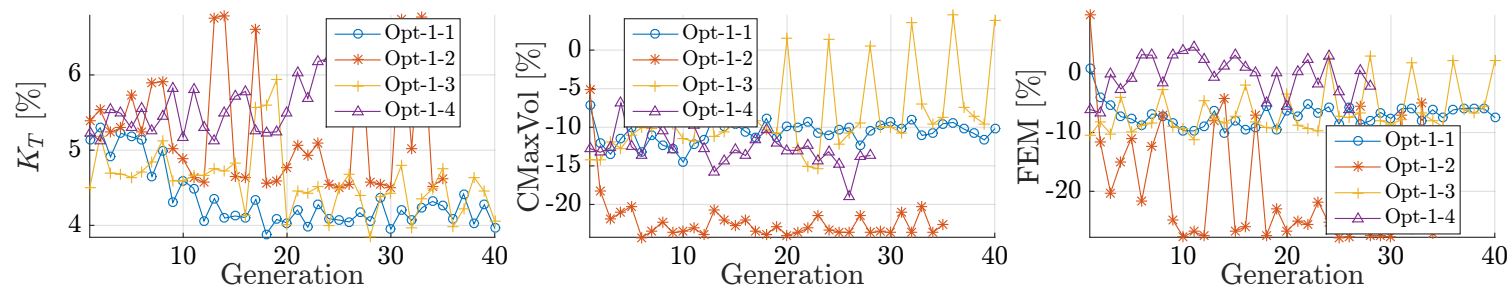

(a) Group 1
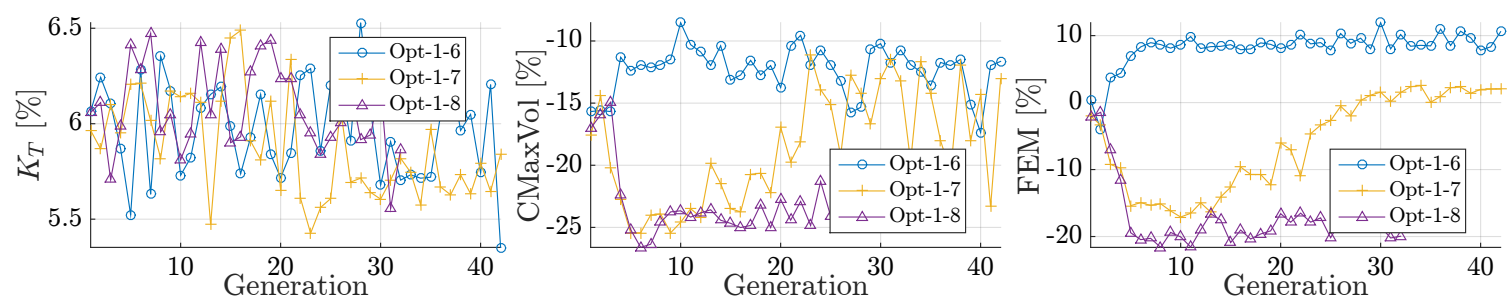

(b) Group 2

Figure 3. Development of the constraints, given as the generation median of $K_{T}$, maximal cavity volume and blade strength, relative to baseline design; Opt-1. (a) Group 1; (b) Group 2. 


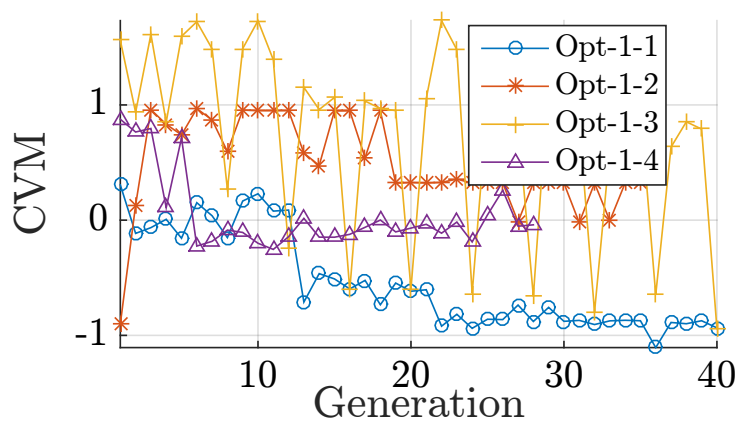

(a) Group 1

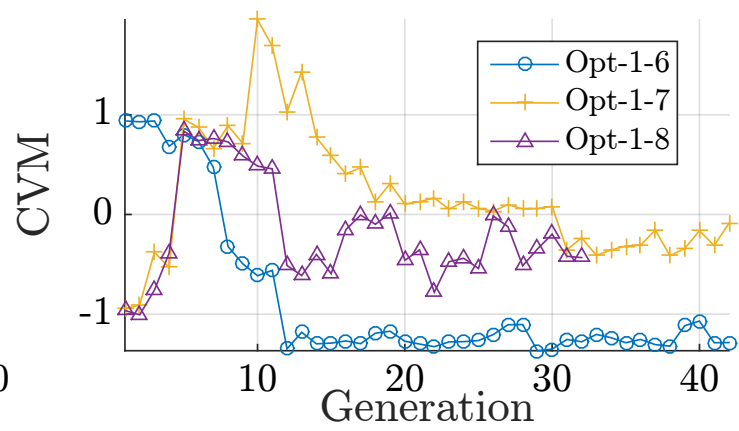

(b) Group 2

Figure 4. Development of the feasibility, given as the generation wise validity ratio and the generation median of Constraint Violation Measure (CVM). (a) Group 1; (b) Group 2.

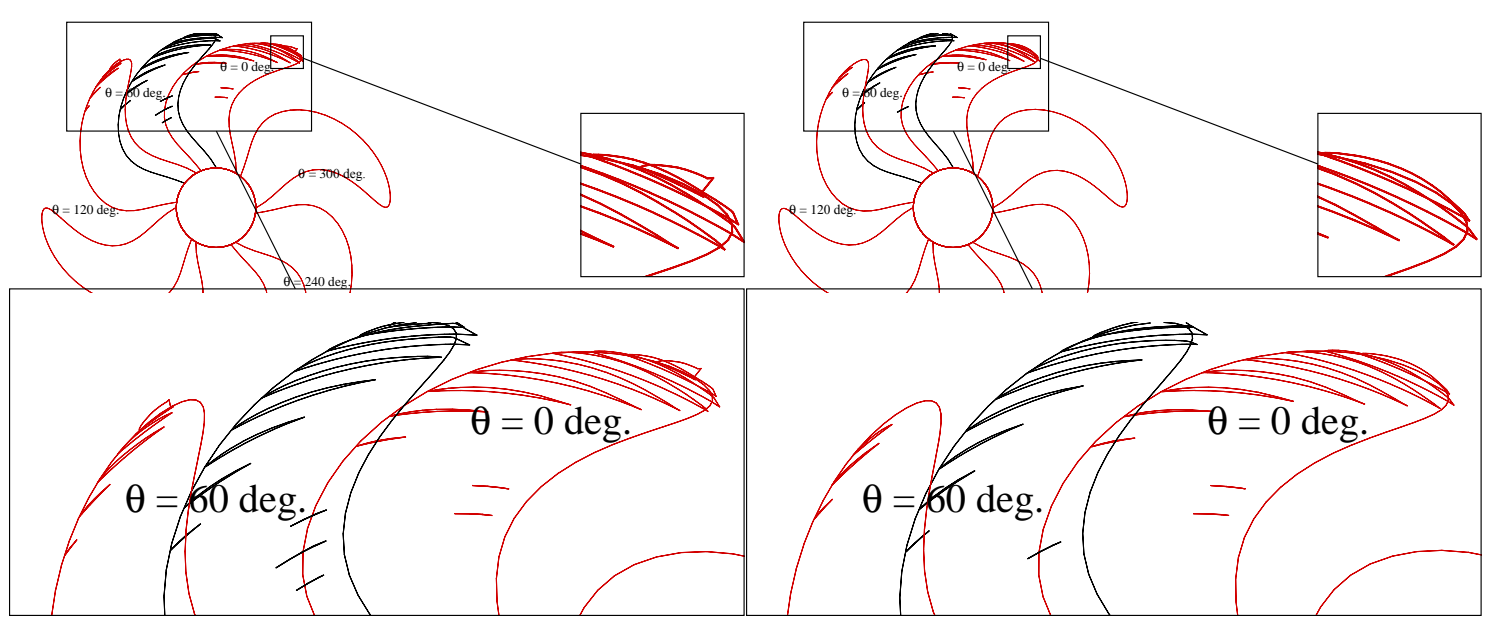

(a) Opt-1-6

(b) Opt-1-7

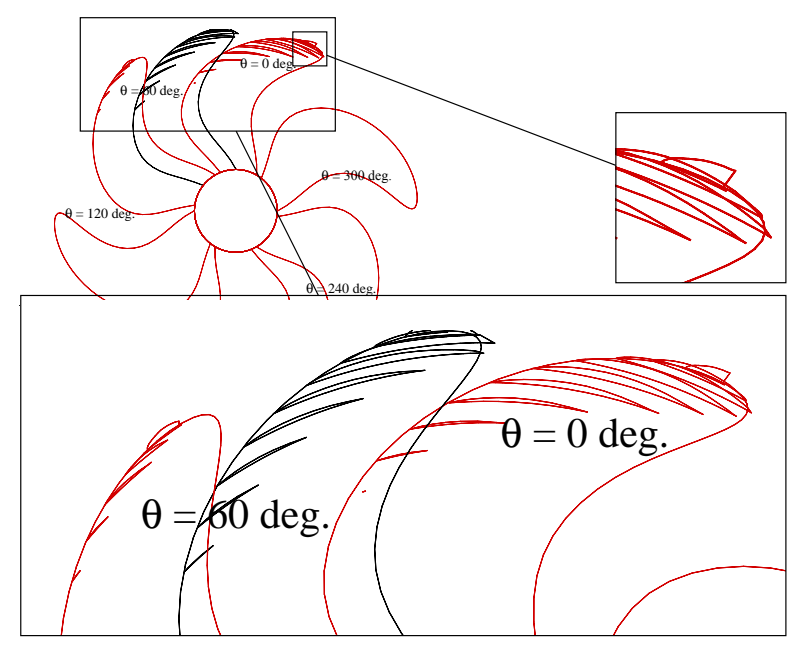

(c) Opt-1-8

Figure 5. Cavitation performance of propeller case Opt-1, Group 2. (a) Opt-1-6; (b) Opt-1-7; (c) Opt-1-8.

For the final comparison with the benchmark design, we select one solution from all Pareto fronts that satisfies the constraints and provide the best trade-off among the objectives to compare with. For Opt-1, the solution is obtained by the PSO algorithm in Group 1, with $0.83 \%$ higher 
efficiency, $14.14 \%$ lower pressure pulses and $32.56 \%$ smaller cavitation volume than the benchmark design. The maximal blade stresses are maintained on the same acceptable level as the benchmark propeller $(+0.18 \%)$. In Figure 6 the simulation results are compared of both designs. The optimisation utilising only parametrised geometrical curves is successfully conducted and yields a design solution comparable with the manual work. Only the blade tip is different compared to the benchmark propeller which features a particular tip design; the sectional shape is modified to decrease the tip leading at the design point and suppress tip vortex inception. This requires manual interaction in the design process and is based on the designer's experience. Due to the absence of an analysis tool for the tip vortex inception, the optimisation yields a higher loading at the tip. Figure 6a indicates, however, a smaller sheet cavitation at the outermost radius. The main conclusions regarding Opt- 1 are summarised in Table 5.

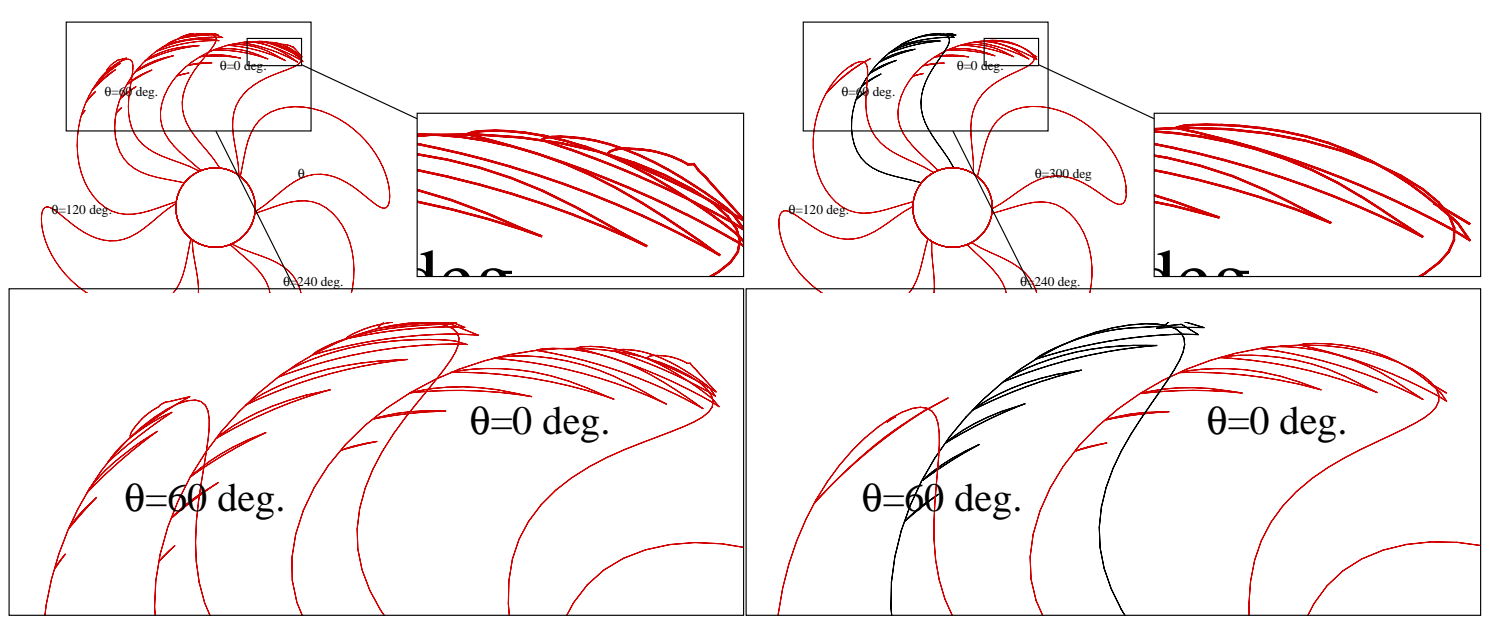

(a) Opt-1 benchmark

(b) Opt-1-2

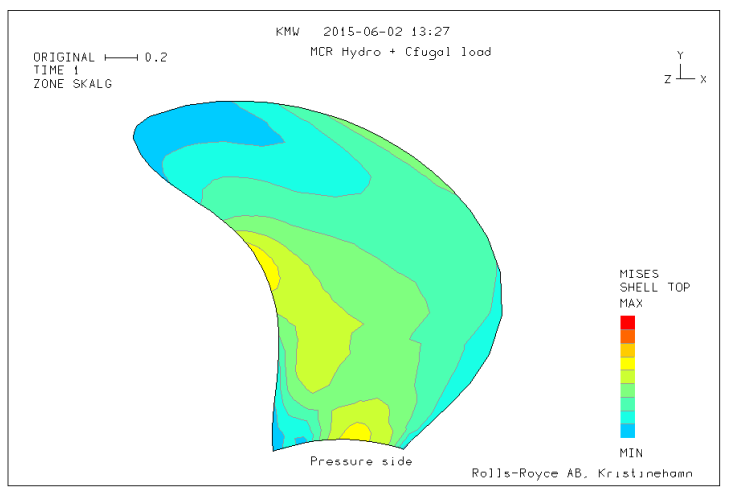

(c) Opt-1 benchmark

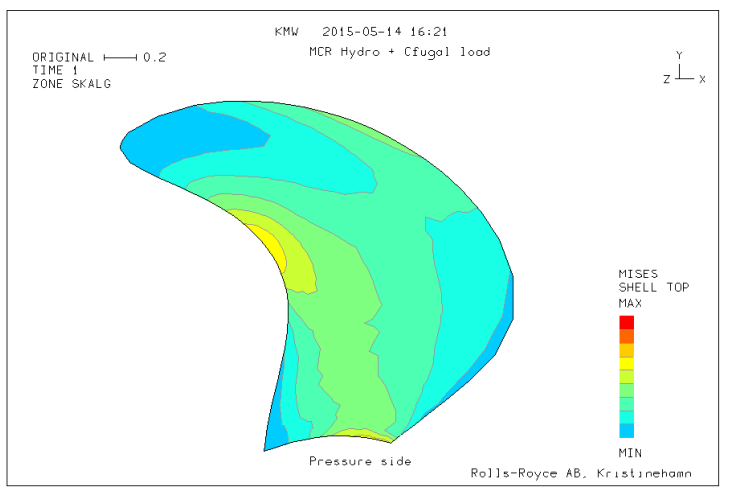

(d) Opt-1-2

Figure 6. Cavitation performance of a finite element method (FEM) simulation of hydrodynamic and centrifugal loads of benchmark propeller case Opt-1 and optimised design Opt-1-2. (a) Opt-1 benchmark; (b) Opt-1-2; (c) Opt-1 benchmark; (d) Opt-1-2. 
Table 5. Potential improvement and convergence observations of Opt-1.

\begin{tabular}{|c|c|}
\hline Group & Observation \\
\hline I & $\begin{array}{l}\text { - Opt-1-3 (AS NSGA-II) best objective improvement ( } \eta \geq 1 \%, p \leq-5 \% \text { ), yet too optimistic. } \\
\text { Median of objectives spikes at every fourth generation with calculated evaluations (Figure A1a). } \\
\text { Yet meta-model adapts. } \\
\text { - Opt-1-2 (PSO) and Opt-1-4 (SANA NSGA-II) both achieve objective median of about } 0.2 \% \text { to } \\
0.5 \% \eta \text { increase and around } 5 \% p \text { reduction. Yet, } p \text { is not converged. } \\
\text { - Opt-1-1 (NSGA-II) yields highest trustworthy median } \eta \text { increase of about } 0.8 \% \text { with maintained } \\
\text { - } p \text { level. } \\
\text { - Opt-1-1 yields the smallest variation between the generational median of both objectives. } \\
\text { Opt-1-2 and Opt-1-4 vary stronger in pressure pulse objective, which coincides with a variation } \\
\text { of the blade tip loading (Figure A1). } \\
\text { Opt-1-4 varies parameters for root unloading and EAR (Figure A1a). Both EAR and unloading } \\
\text { factors correlate: a reduction of EAR coincides with a higher loaded blade and thereby balances } \\
\text { the efficiency. }\end{array}$ \\
\hline II & $\begin{array}{l}\text { - NSGA-II (Opt-1-6) superior objective capabilities (Figure A1b) but fails regarding constraint } \\
\text { feasibility. } \\
\text { - Opt-1-7 is similar capable in finding good objectives, however, not better with regard to the } \\
\text { constraint feasibility. } \\
\text { - Opt-1-8 (CCent constraint) is the only set up that manages to improve all three objectives and } \\
\text { constraint feasibility, Figures A1 and } 4 \text {. } \\
\text { - No enhanced convergence, although less input parameters reduce the design space. } \\
\text { and no reduction blade weight (Figure A1). }\end{array}$ \\
\hline
\end{tabular}

\subsubsection{Opt-2}

The geometry of Opt-2 (both in Groups 1 and 2) evolves similarly for the majority of cases towards a higher EAR, Skew, Rake and root unloading, Figure 7. Only two parameters vary during the continuation of Opt-2-2: thickness and tip unloading, Figure 7a, which strengthen the assumption of low importance of these parameters. Hence, in Group 2 we reduce the number of decision variables down to three by omitting thickness reduction, blade unloading and skew line starting location on the hub (SkLFhub). The sensitivity analysis provide the fourth lowest importance for the root unloading and is therefore omitted in cases Opt-2-6 to Opt-2-9. This consequent reduction of parameters chokes off the optimisation, and the only true improvement in objectives and constraints is obtained by Opt-2-13. Which is conducted subsequent to Opt-2-6 to Opt-2-9 and uses again two more parameters (TMAX and root unloading).

The strongest effect can be assigned to the unloading root factor, by analysing the development of the additional parameter towards a root unloading of up to $50 \%$, Figure $7 \mathrm{~b}$. Neither the tip unloading nor the blade thickness evolves to a significant geometry change. The positive efficiency median (around $0.3 \%$ in Figure A3b), despite larger blade area, can be seen as the result of less rotational losses due to the root unloading, which has not been captured by the sensitivity analysis.

The consequent violation of the $K_{T}$ constraint of Opt-2-1 is evident from Figure 8a. Yet it is not seen by the algorithm since the CVM is positive ( $>1 \%)$. The CVM balances infeasible $K_{T}$ with significantly feasible blade stresses and caviation ( $-10 \%$ in cavitation volume and $-30 \%$ in blade stress). In case of the SANA NSGA-II (Opt-2-4) a sudden change of EAR at generation 16 and the consistent increase in cavitation abrogates the balance and CVM drops consequently, Figure 8a. The development of CVM reveals also that the meta-model of the AS NSGA-II (Opt-2-3) underestimates the constraints which spikes at every fours generation; particularly the stress prediction of the meta-model, deviates from the calculated values by $5 \%$ (Figure $8 \mathrm{a}$ ). 

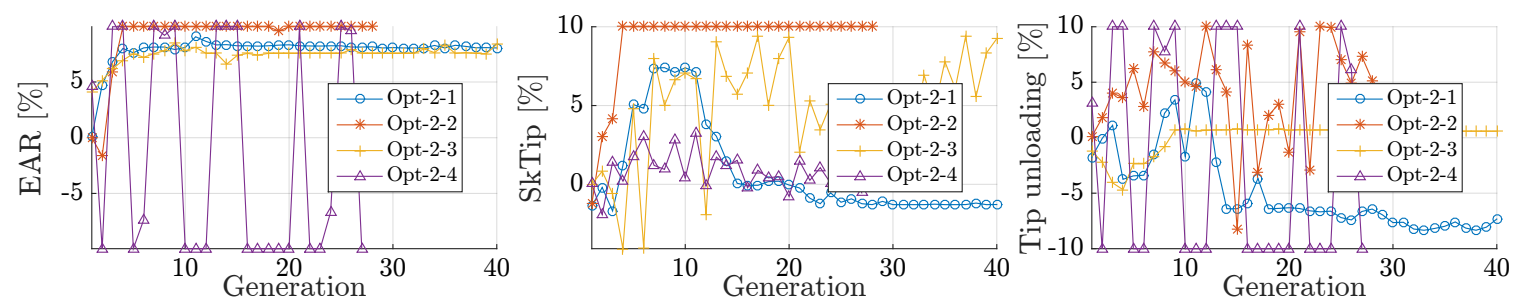

(a)
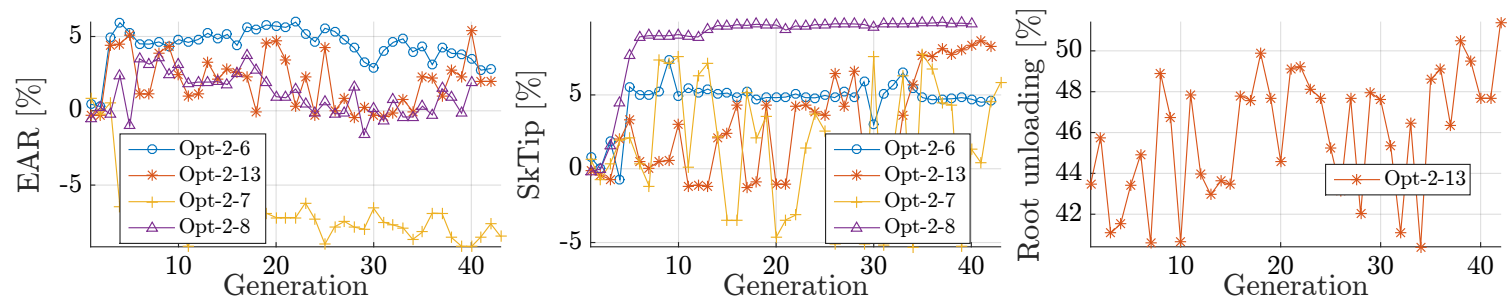

(b)

Figure 7. Parameter development, given as median per generation, relative to baseline design. (a) Selected geometry parameters of optimisations in Group 1; (b) Geometry parameters of optimisations in Group 2.

In Group 2 the $K_{T}$ constraint violation is improved by two of the cases, Opt-2-8 and Opt-2-13 (Figure 8b). Both Opt-2-8 and Opt-2-13 reduce the number of variants violating $K_{T}$, or $K_{T}$ in combination with other constraints, from about $60 \%$ of variants (Opt-2-6 $=60.3 \%$, Opt-2-7 $=69.2 \%$ ) to $17.2 \%$ (Figure 9b) and 34.8\% (Figure 9a) respectively. Opt-2-8 uses cavity shape constraints, which are hardly met or affected by applied geometrical changes, hence $K_{T}$ is the only characteristic that can be modified. Opt-2-13 amplifies the $K_{T}$ constraints and thereby increases its influence on the CVM. The additional parameters, which are utilised in Opt-2-13, are contradictory to an increase in thrust, since the optimisation decreased the blade loading. The improved $K_{T}$ is consequently a result of the amplified constraint handling.
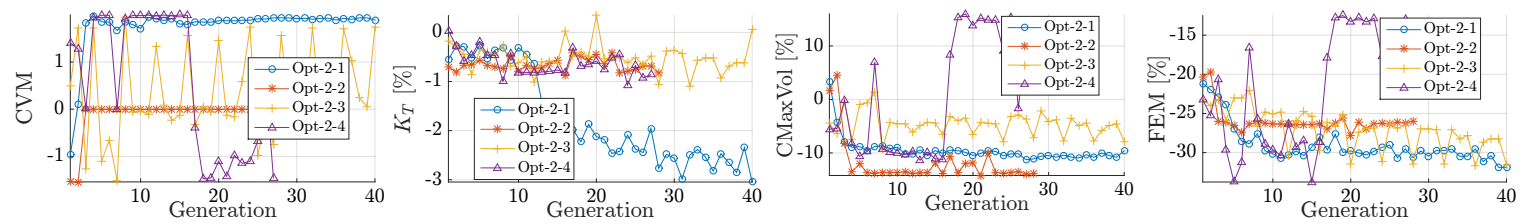

(a) Group 1
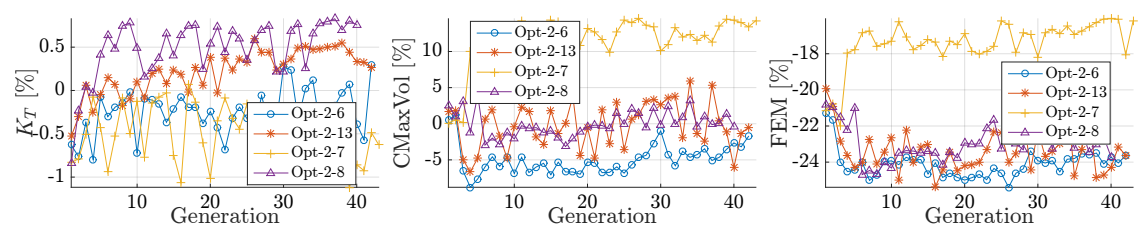

(b) Group 2

Figure 8. Development of constraints in Group 1 (a); and Group 2 (b). 


\begin{tabular}{|l|}
\hline \\
\hline valid \\
$K_{T}$ \\
FEM \\
CLength \\
$K_{T}+$ \\
CavMaxVol, \\
CLength \\
FEM, CavMaxVol, \\
CLength \\
\hline
\end{tabular}

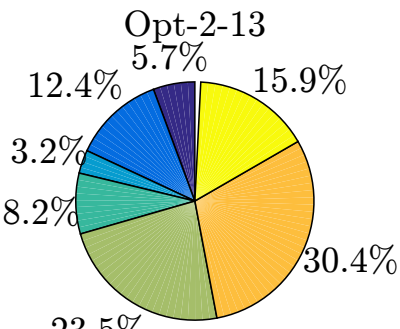

$23.5 \%$

(a)

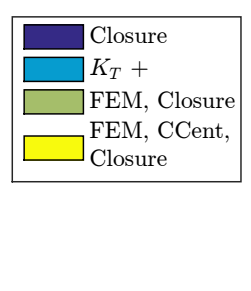

$54.3 \%$

(b)

Figure 9. Analysis of constraint violation considering all created variant per optimisation case.

(a) Opt-2-13; (b) Opt-2-8.

We select a Pareto optimal solution from Opt-2-13 for the the detailed analysis of cavitation on propeller Opt-2 (Figure 10). The sheet cavity volume of the baseline is substantial at the $\theta=0^{\circ}$ position and increases at the blade tip in thickness when the blade passes the $\theta=30^{\circ}$ position. Hence, two constraints are expected to improve the cavitation, the constraints on (i) maximal cavitation volume; and (ii) on cavitation sheet thickness at the tip. Opt-2-6 achieves with the CMaxVol constraint the best median improvement of $-5 \%$ (Figure $8 \mathrm{~b}$ ). The Pareto optimal solution is, however, less improved $(-0.12 \%$, Table 6$)$. The cavity thickness constraint fails in Opt-2-7 primarily due to a lack of sufficient parameters to modify the blade geometry locally at the tip and therefore only the cavity change constraint affects the design during the optimisation by reducing $p$ (Table 6 ), which is the only case that achieves a reduction in pressure pulse together with increased cavity volume. Opt-2-8 enables cavity centroid constraints which, again, occurs most harmful towards the tip region where the parameters are only marginally effective.

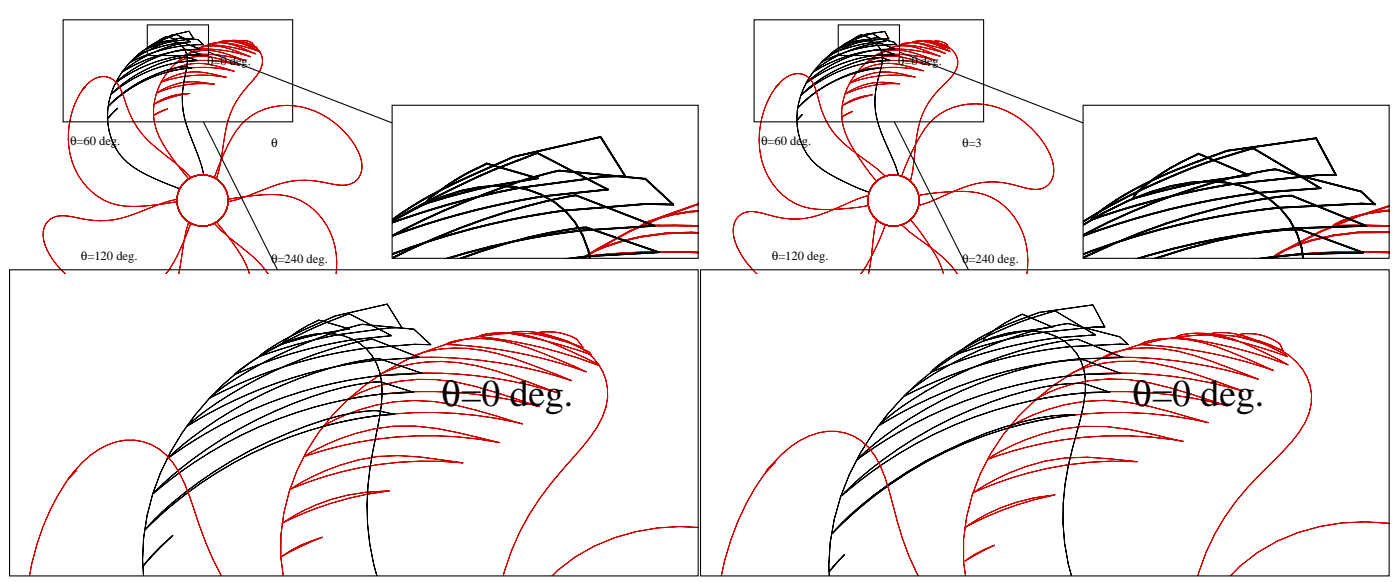

(a) Opt-2 baseline

(b) Opt-2-13

Figure 10. Cavitation performance of propeller case Opt-2; suction side sheet cavitation prediction by MPUF-3A. (a) Opt-2 baseline; (b) Opt-2-13.

Table 6. Selected optimal solutions relative to baseline design.

\begin{tabular}{ccccccc}
\hline Optimisation & Variant & $\eta[\%]$ & $p[\%]$ & CavVol [\%] & FEM [\%] & $K_{T}[\%]$ \\
\hline Opt-2-6 & 602 & 0.39 & 0.24 & -0.12 & -22.08 & -0.24 \\
Opt-2-13 & 896 & 0.47 & -3.75 & -5.34 & -31.06 & -0.31 \\
Opt-2-7 & 600 & 0.32 & -0.37 & 1.92 & -19.11 & -0.36 \\
Opt-2-8 & 939 & 0.47 & 1.72 & 1.89 & -22.08 & -0.31 \\
\hline
\end{tabular}


In Group 3 we focus on the performance improvement which is achieved according to Figure A3c by local modification of pitch at $r / R=0.6$ and $r / R=1.0$, chord length at $r / R=0.5$, camber at $r / R=0.8$ and skew at $r / R=1.0$. The analysis of constraints reveal that the CVM is in fact positive for all three cases in Group 3, yet the efficiency gain is only achieved by violating the required $K_{T}$ (Figure 11b). The CVM balances with considerable improvements in blade stress (Figure 11d) and cavity volume (Figure 11c).

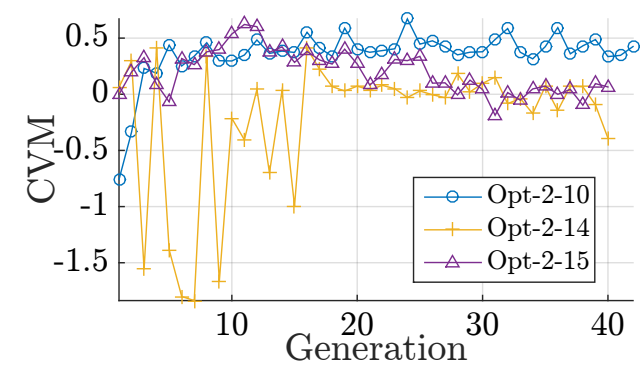

(a) CVM

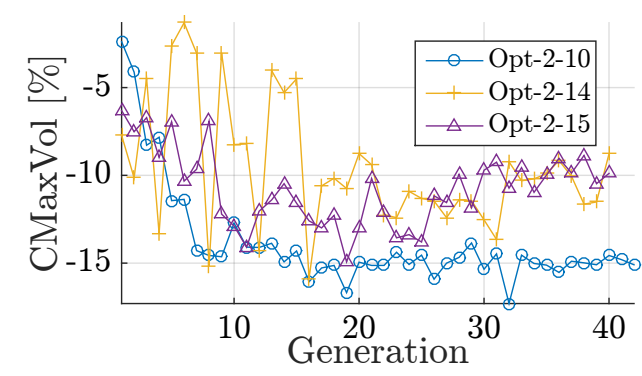

(c) Cavity volume

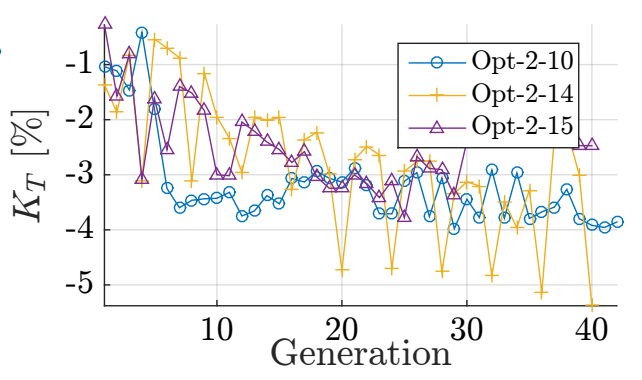

(b) $K_{T}$

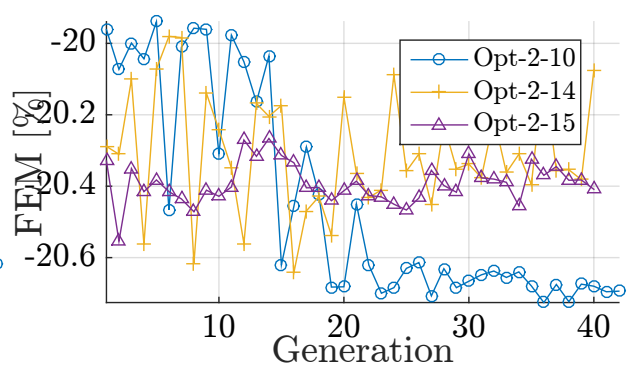

(d) FEM

Figure 11. Development of constraints in Group 3. (a) CVM; (b) $K_{T}$; (c) Cavity volume; (d) FEM.

The performance of selected solutions in Group 3 which provide improvements in objectives and constraints and primarily fulfil the required $K_{T}$ are given in Table 7, relative to the performance of the benchmark design. All three algorithms in Group 3 yield the same level of improvement in objectives, blade stress and cavitation reduction and can be considered to achieve the same optimisation quality. This highlights an advantage of Opt-2-14, which utilises the adaptive meta-model, and reduces the lead time by a factor of five. The variations in constraints due to the difference between estimations and calculations are high at the beginning (Figure A3c), but shrink with improving meta-model, and yielding the same geometry as employing only calculated results. The main conclusions regarding Opt-2 are summarised in Table 8.

Table 7. Optimal solutions of Group 3, relative to performance of manual design.

\begin{tabular}{ccccccc}
\hline Optimisation & Variant & $\eta[\%]$ & $p[\%]$ & CavVol [\%] & FEM [\%] & $K_{T}[\%]$ \\
\hline Opt-2-10 & 399 & 0.98 & -17.33 & -26.39 & -23.86 & -0.97 \\
Opt-2-14 & 622 & 1.50 & -14.34 & -22.85 & -23.59 & -0.90 \\
Opt-2-15 & 638 & 1.03 & -20.50 & -26.97 & -23.69 & -0.84 \\
\hline
\end{tabular}


Table 8. Potential improvement and convergence observations of Opt-2.

\begin{tabular}{|c|c|}
\hline Group & Observation \\
\hline I & $\begin{array}{l}\text { - Small improvement potential in efficiency }(\leq 0.2 \%),-10 \% \text { in } p \text { (Figure A3). } \\
\text { - Opt-2- } 1 \text { and Opt-2-3 yield similar } \eta \text { and } p \text { improvements }(\eta \approx 0.2 \%, p \leq-5 \%) \text {. } \\
\text { - Discrepancy between meta-model of Opt-2-3 and calculated results } \leq 3 \% \text { in } p \text {. } \\
\text { - Opt-2-2 emphasises on pressure pulse reduction. }\end{array}$ \\
\hline II & $\begin{array}{l}\text { - Less improvement possible compared with optimisation of Group } 1 . \\
\text { - Only Opt-2-13 able to improve both objectives. }\end{array}$ \\
\hline III & - Substantial performance gain $(\eta>0.6 \%$ and $p<-5 \%)$, Figure A3c. \\
\hline
\end{tabular}

\subsubsection{Opt-4}

The underestimation of pressure pulses in both cases that use the AS NSGA-II (Opt-4-3 and Opt-4-14) yields a thickness reduction which is opposed to the other algorithms. From Figure 12b it becomes particularly obvious that, at generations 4 and 8, and from Figure 12a at generation 4, a thickness reduction yields a positive effect on the objective (Figure A5a,c) and in both cases initiate the lasting trend for the thickness reduction.

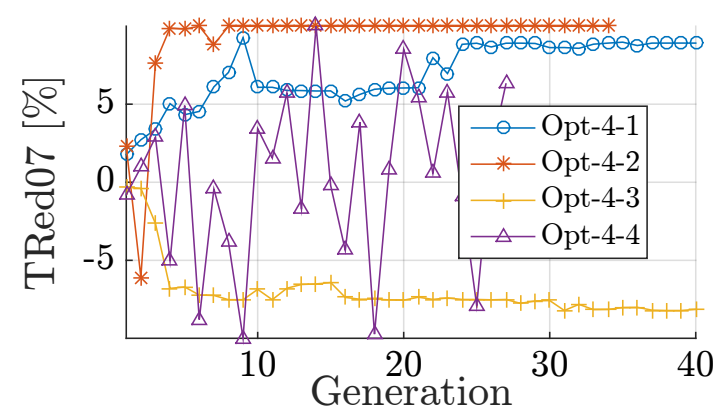

(a) Group 1

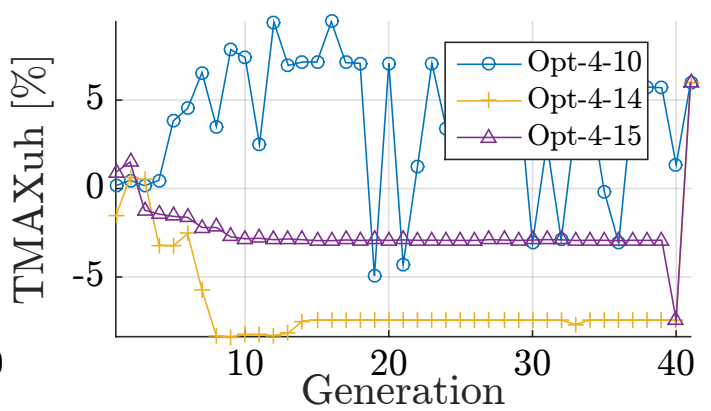

(b) Group 3

Figure 12. Development of blade thickness parameter at $r / R=0.7$ as median per generation, relative to baseline design. (a) Group 1; (b) Group 2.

The input parameters are reduced in Group 2 by all three pitch parameters (unload factor root, unload factor tip and unload width). Their effect on constraints, objectives and convergence is marginal which is consistent with the result of a sensitivity analysis, employing the extendedFAST method by Saltelli and Bolado [24] (Figure 13). The importance measure is given as an aggregated sensitivity measure of the sensitivity indices of each objective and constraint. The unloading importance is assigned low which results from a varying unloading importance among the objective and constraints; both unloading parameters affect the cavitation rather than the efficiency. However, the span wise distribution of unloading (Unload-Width) is more important for the effect on efficiency than for the cavitation. This explains why the method assigned a higher importance to the unload width when the unloading itself is assigned less important. There are two more parameters, the skew line position on the hub and the rake, which are assigned with low importance, however, we included both in Group 2, since the skew at the tip is assigned with high importance and an effect of rake on the efficiency is noted in other cases. 


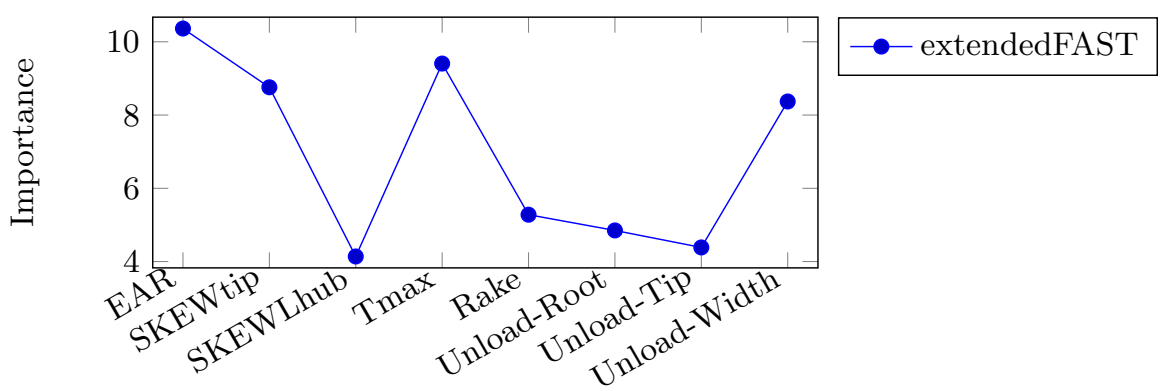

Figure 13. Importance of input parameter according to sensitivity analysis.

The results of Group 2 show, by strict convergence of rake, that this parameter is of importance for the obtained performance. However, for the performance increase in Group 3, we initially applied modifications of pitch and camber at $r / R=0.7$ and $r / R=1.0$ and of chord length at $r / R=0.6$, which resulted in incapable performance (Figure 14a). The adoption of an additional chord length and the thickness parameters improved the performance (Figure 14b), yet only the inclusion of rake and skew at the tip modifications yield extended improvements (Figure 14c). This is consistent with the result of the sensitivity analysis.

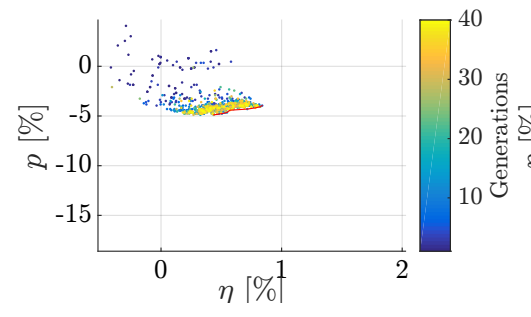

(a)

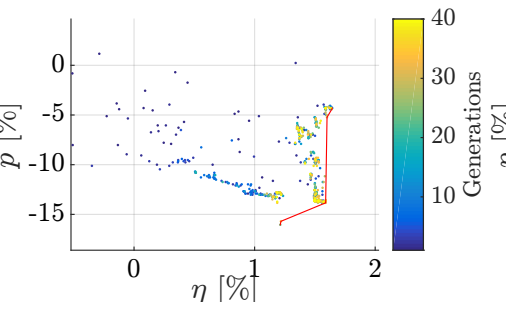

(b)

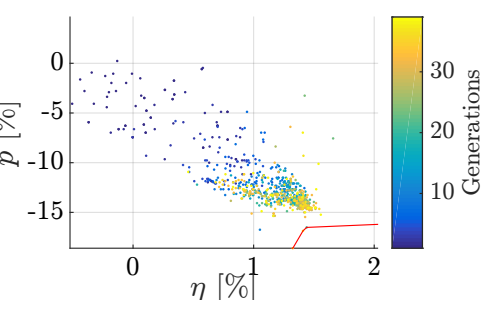

(c)

Figure 14. Pareto front in objective space for initial set-ups of opt-11-10 (a,b) and final set-up (c).

The margin for constraints violation is sufficiently large for Opt-4. The $K_{T}$ constraint is feasible according to the definition as an inequality constraint and the optimisations adapt from a too high $K_{T}$ towards the desired value of the baseline (Figure 15a). The stress constraint is similarly met and improved during the optimisation by $-10 \%$ to $-20 \%$ Figure $15 \mathrm{a}$.

Comparing the baseline design with the manually designed benchmark propeller reveals $2 \%$ higher efficiency for the benchmark design, which is a significant difference between the baseline and benchmark design. The manual work on the design increased the performance which is similarly achieved only by one of the Pareto optimal solutions (Opt-4-10), featuring an increase in cavitation volume. However, even if the optimisations achieve pressure pulse reduction of up to $18 \%$, compared to the baseline, they are still 3\% higher than the benchmark design. The solution discovered by the optimisation is not quite as optimal as the manually designed blade. This is possibly the consequence of too narrow box limits on the selected parameters, since they converged in all cases rapidly to the limits and a baseline design which is far too off to provide the required performance. However, the design of the support optimisation is close to the benchmark design with $0.23 \%$ efficiency increase and $21.6 \%$ reduction in blade stress, compared to the benchmark. The main conclusions regarding Opt- 4 are summarised in Table 9. 


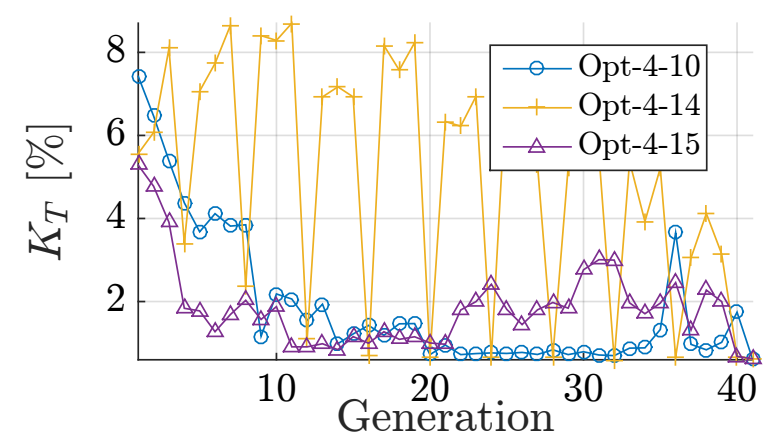

(a) $K_{T}$

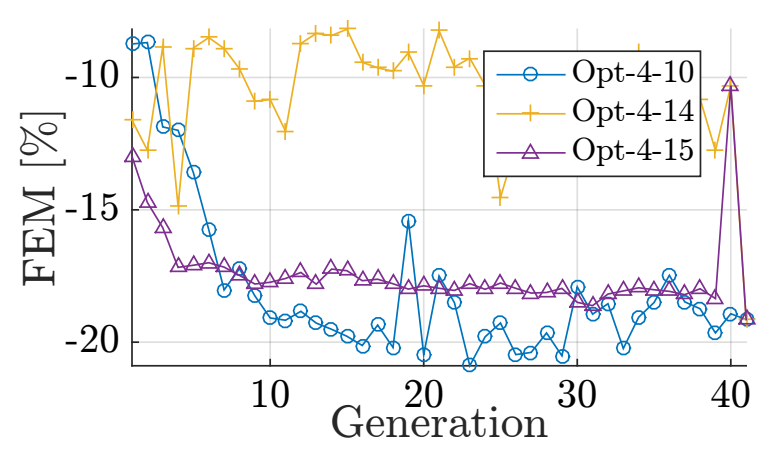

(b) FEM

Figure 15. Development of $K_{T}(\mathbf{a})$ and FEM constraints (b) in Group 3.

Table 9. Potential improvement and convergence observations of Opt-4.

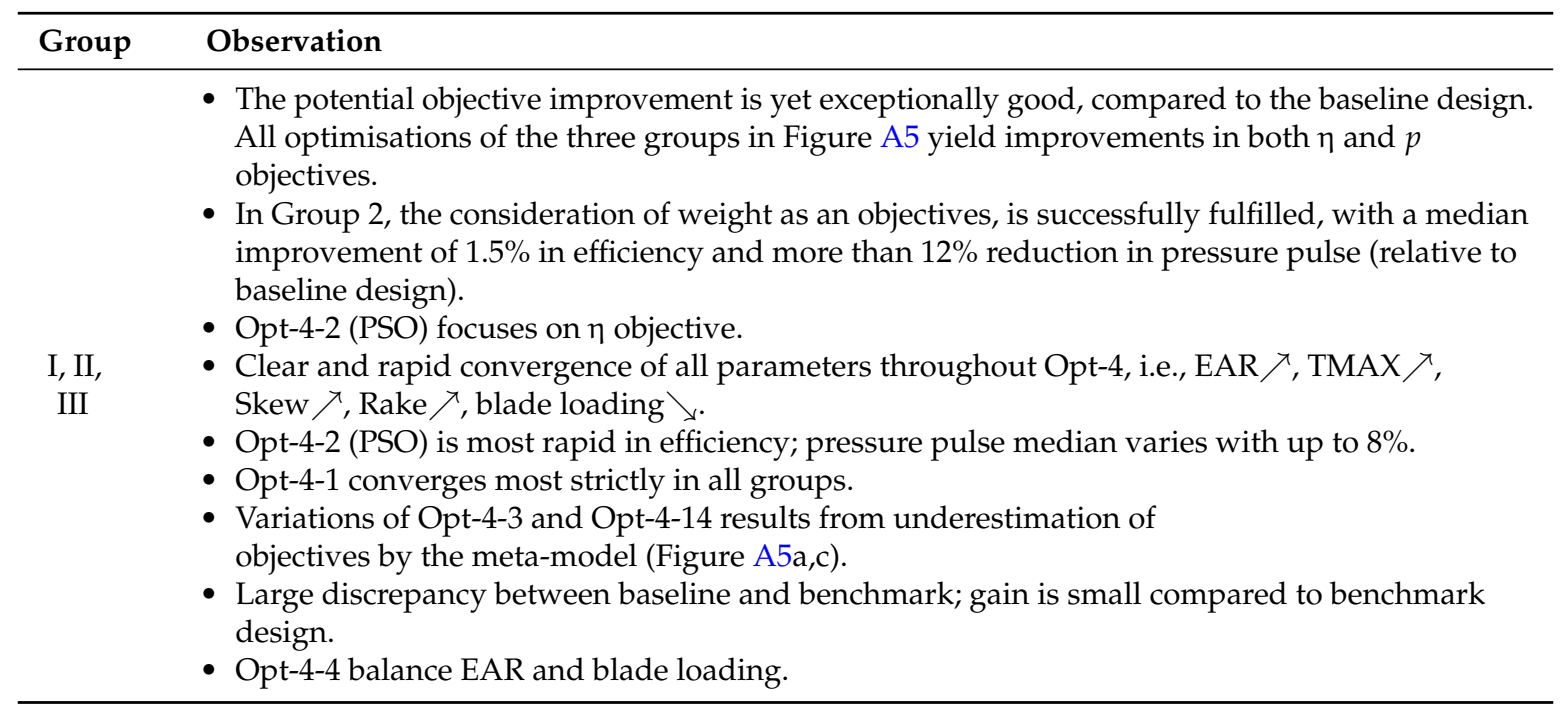

\subsection{Constraint Optimisation}

\subsubsection{Opt-3}

Assessment of optimisation results have extensively been discussed in [13] and therefore only a brief summary is provided here. Several propeller alternatives originate from the baseline design and were tested in model tests as part of an internal design study. The results confirm that this design is all about to balance the blade loading such that tip vortex strength decreases, but erosive mid-chord cavitation is still contained.

The cavitation constraints and handling of constraints by amplification in Group 2 show that optimisations with enabled cavity shape constraints (Opt-3-7 to Opt-3-9), result in an increased number of solutions with lower pressure pulses. Additionally, it is possible to control the cavity shape and the objectives with the proposed constraints such that the optimisation yields different geometries. For instance, an amplification of cavitation length yields a reduction of the cavitation length median of $2 \%$ which on the other hand increases pressure pulses due to a constant cavity volume. Opt-3-8, with constraint on cavitation centroid, yields feasible solutions with a centroid relocated upstream by increasing the cavitation at the leading edge (Figure 16b). Optimisation case Opt-3-9 also utilises an amplified $K_{T}$ constraint and thereby enables a drop in $K_{T}$ at generation 6 (Figure 17b), down to the baseline value which eventually allows for raising efficiency between generation 1 and 10 (Figure 17a). 


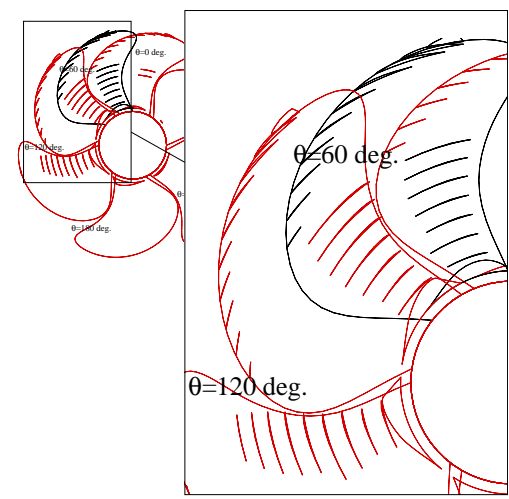

(a) Opt-3 baseline

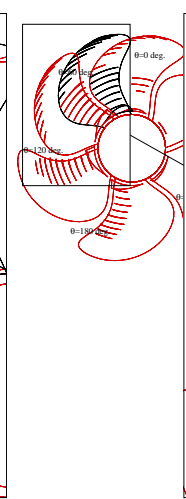

(b) Opt-3-8

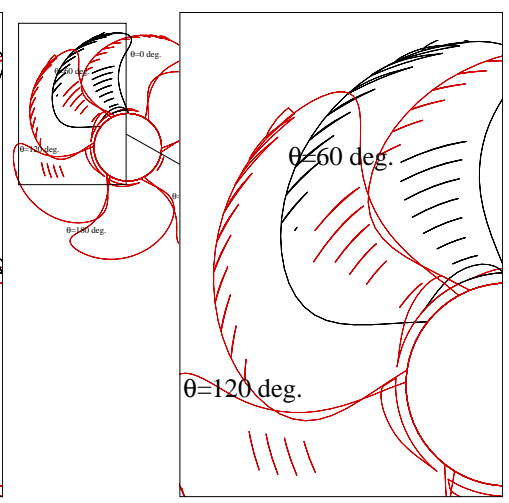

(c) Opt-3-15

Figure 16. Cavitation prediction of propeller Opt-3. (a) Opt-3 baseline; (b) Opt-3-8; (c) Opt-3-15.

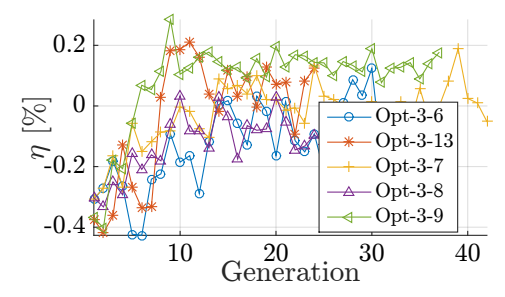

(a) Median $\eta$

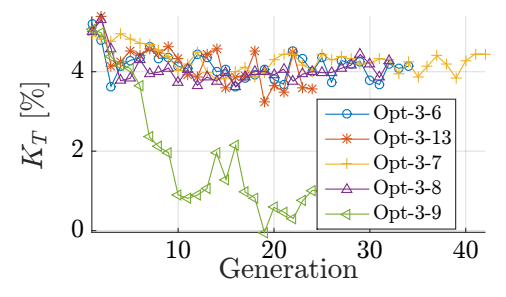

(b) Median $K_{T}$ constraint

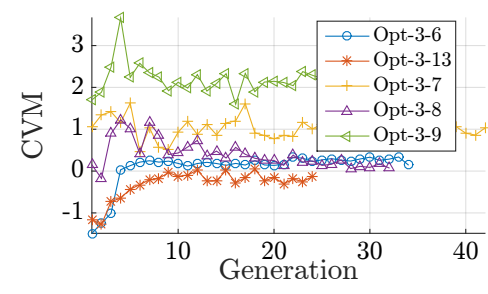

(c) CVM Opt-3

Figure 17. Influence of $K_{T}$ amplification in Opt-3-9. (a) Median $\eta$; (b) Median $K_{T}$ constraint; (c) CVM Opt-3.

Local modification are applied primarily to improve the cavitation. The results show that the selection of parameters, based on the location of cavities, and the results of a sensitivity analysis, yield the desired cavitation improvement and maintenance of the objectives. The optimal solutions are remarkably similar among Group 3, except for a difference in thickness. This highlights an advantage of the AS NSGA-II (opt-3-14), which reduced the lead time by a factor of 5, again. The SANA NSGA-II requires approximately the same computational time as the NSGA-II, yet achieves the best cavitation prediction (Figure 16c).

The Pareto optimal solutions of Group 3 are comparable with the manually designed benchmark propeller. The best trade-off design is achieved by Opt-3-15 which provides an increase in efficiency, reduction of pressure pulses but still a higher maximal cavitation volume, Table 10. The cavitation of this design (Figure 16c) is in fact distributed over a broader blade section, yet it is thinner compared to the manual design (Figure 18a), hence, contains less focusing energy and is therefore considered to offer a higher margin for cavitation erosion. Beneficial is also the stress distribution over the blade for Opt-3-15 design since it avoids higher peaks at the root (Figure 18). The main conclusions regarding Opt-3 are summarised in Table 11. 
Table 10. Pareto optimal solutions of Group 3, relative to performance of manual design.

\begin{tabular}{cccccc}
\hline Optimisation & Variant & $\eta[\%]$ & $p[\%]$ & CavVol [\%] & $\boldsymbol{K}_{T}[\%]$ \\
\hline Opt-3-10 & 183 & 1.77 & -1.22 & 64.25 & 1.23 \\
Opt-3-14 & 755 & 1.81 & -1.05 & 66.47 & 1.29 \\
Opt-3-15 & 185 & 1.79 & -1.38 & 59.81 & 0.97 \\
\hline
\end{tabular}

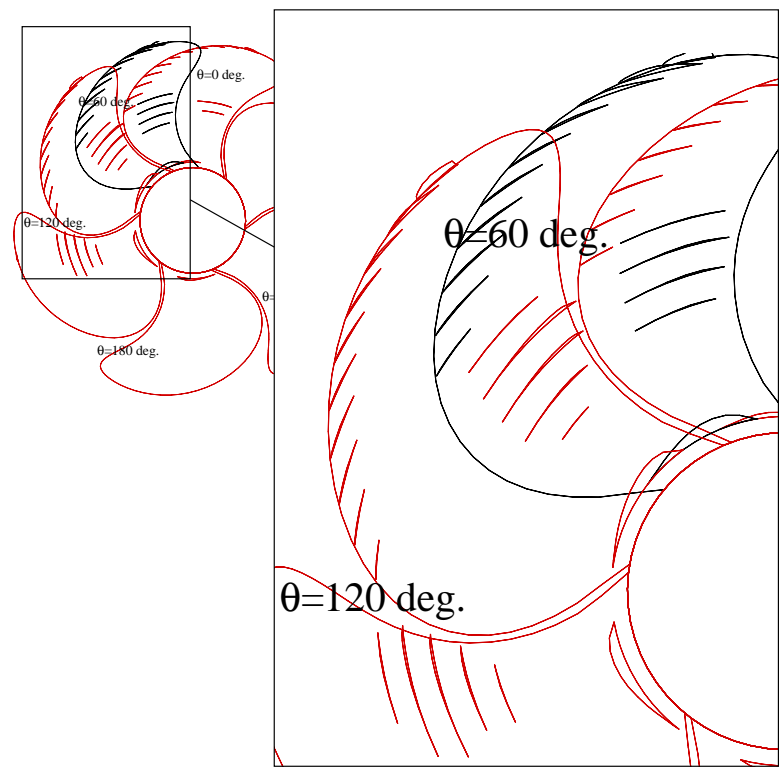

(a) Opt-3 benchmark

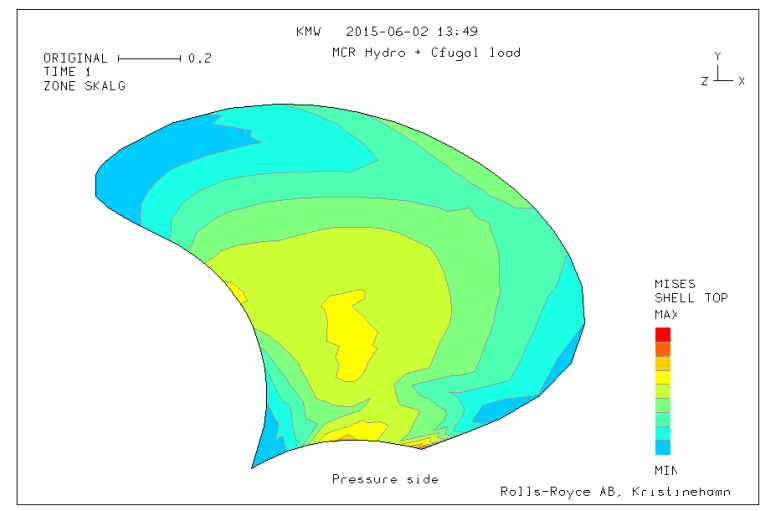

(b) Opt-3 benchmark

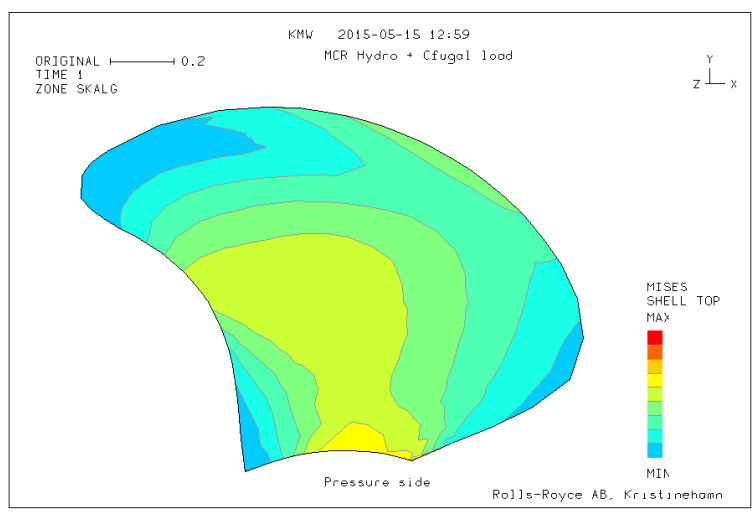

(c) Opt-3-15

Figure 18. Cavitation performance (a) and FEM simulation of hydrodynamic and centrifugal loads of benchmark propeller case Opt-3 (b) and optimised design (Opt-3-15) (c). 
Table 11. Potential improvement and convergence observations of Opt-3.

\begin{tabular}{|c|c|}
\hline Group & Observation \\
\hline I & $\begin{array}{l}\text { - Potential improvement between the algorithms diverge, with an absolute difference of } \\
1 \% \text { in } \eta \text { and } 8 \% \text { in } p \text {. } \\
\text { - Opt-3-1 (NSGA-II) and Opt-3-3 yield no increase in } \eta \text { and only } 2 \% \text { reduction of } p \text {. } \\
\text { - Opt-3-4 and Opt-3-2 explore the objective space broadly distributed and achieve } \\
\text { median improvements of } \eta=1 \% \text { and } p=-10 \% \text {. } \\
\text { - PSO (Opt-3-2) pushes the Pareto front even furthest towards high } \eta \text { of upto } 0.9 \% \text { and } \\
\text { low } p=-13 \% \text {. } \\
\text { - Opt-3-4 and Opt-3-2 converge towards a desired Pareto front, rapidly and provide } \\
\text { after } 10 \text { generations a positive CVM. } \\
\text { - Meta-model in Opt-3-3 underestimates the constraints. }\end{array}$ \\
\hline II & $\begin{array}{l}\text { - Performance potential improved compared to NSGA-II in Group } 1 \text { (Opt-3-1): } \\
0.1 \%-0.2 \% \text { higher } \eta \text {. } \\
\text { - The reduction of decision variables does not yield a positive effect on the number of } \\
\text { variants needed to convergence. }\end{array}$ \\
\hline III & $\begin{array}{l}\text { - Median } \eta \text { improvement highest: } 0.2 \%-0.3 \% \text {. } \\
\text { - Median } p \text { increases up to } 0.2 \% \text {. } \\
\text { - All algorithms perform similarly. }\end{array}$ \\
\hline
\end{tabular}

\subsubsection{Opt-5}

Figure 19a provides that feasible CVM medians are achieved in Group 1 by PSO optimisation (Opt-5-2) and to a large extent for the NSGA-II (Opt-5-1) optimisation. The PSO convergences rapidly within 6 generations and provides a CVM which is annulled and thereby feasible. In case of SANA NSGA-II (Opt-5-4), it is obvious that the large increase in efficiency is traded for feasibility. A violation of the thrust coefficient $K_{T}$ is, however, present in almost all optimisations and where CVM balance mainly with feasible blade stress constraint but partly by a feasible cavitation volume (e.g., Opt-5-2).

No constraint amplification and no cavity shape constraints (Opt-5-6) results in an entirely infeasible set of solutions, mainly violating the required thrust constraint in combination with other constraints on the cavity (94.1\%). The CVM is yet still positive (Figure 19b) due to improvements in blade stresses (Figure 20b). Cavitation, however, is violated which can be seen from the constraints development in Figure 20a towards a median increase of about $140 \%$. In Figure 21a a solution is presented that improves the objectives and violates the $K_{T}$ with only $-0.36 \%$. Yet a cavity volume increase is evident at the root and over the blade surface as mid-chord cavitation.

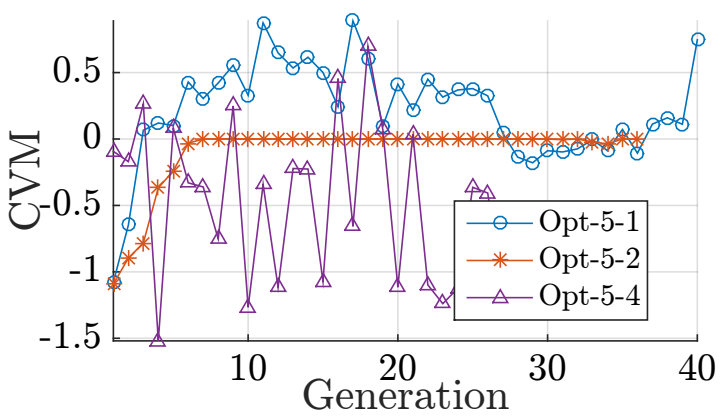

(a) Group 1

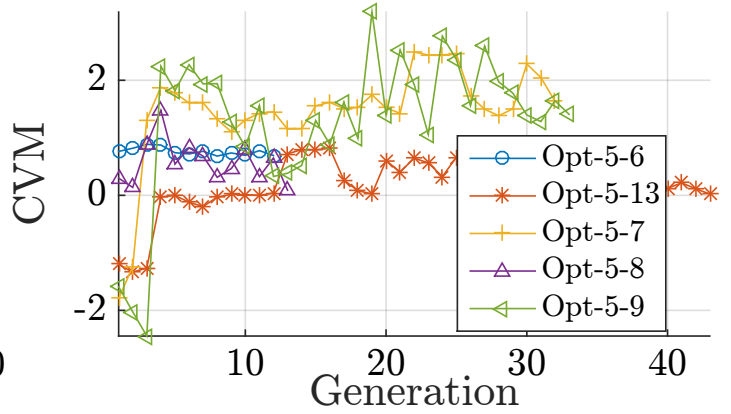

(b) Group 2

Figure 19. Development of the feasibility, given as the generation wise validity ratio and the generation median of CVM. (a) Group 1; (b) Group 2. 


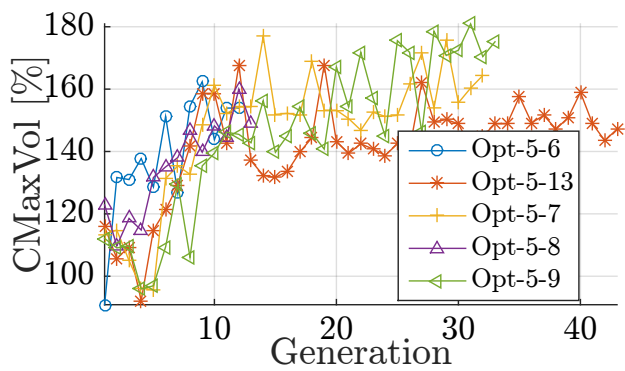

(a)

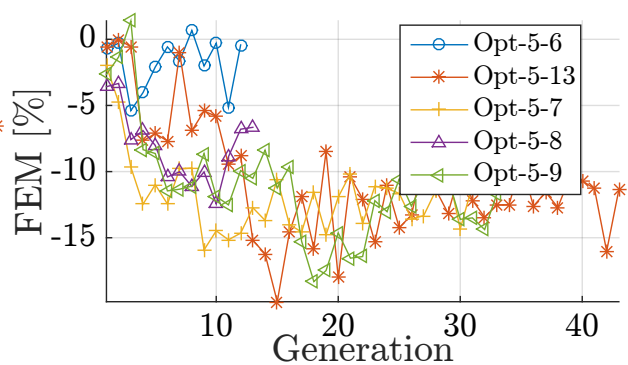

(b)

Figure 20. Development of the constraints, given as the generation median relative to baseline design. (a) Cavitation volume constraint of Opt-4 (Group 2); (b) FEM constraint of Opt-4 (Group 2).

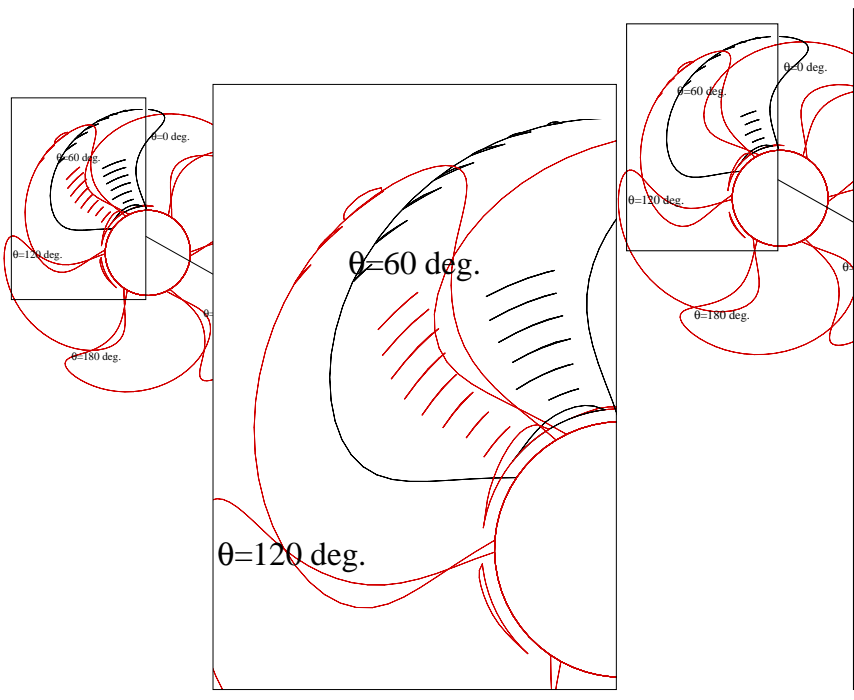

(a) Opt-5-6

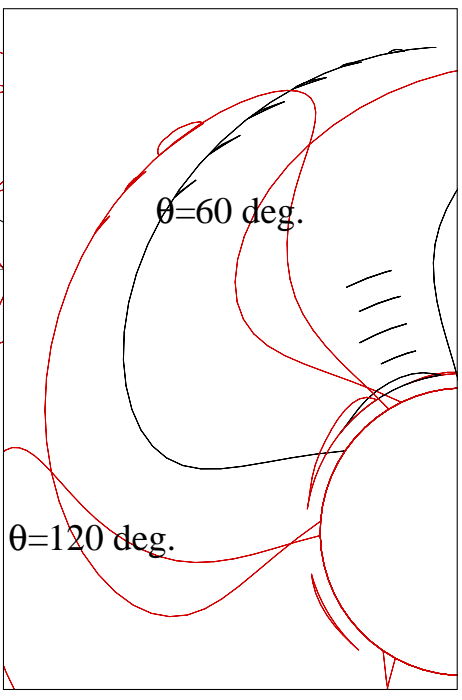

(b) Opt-5-13

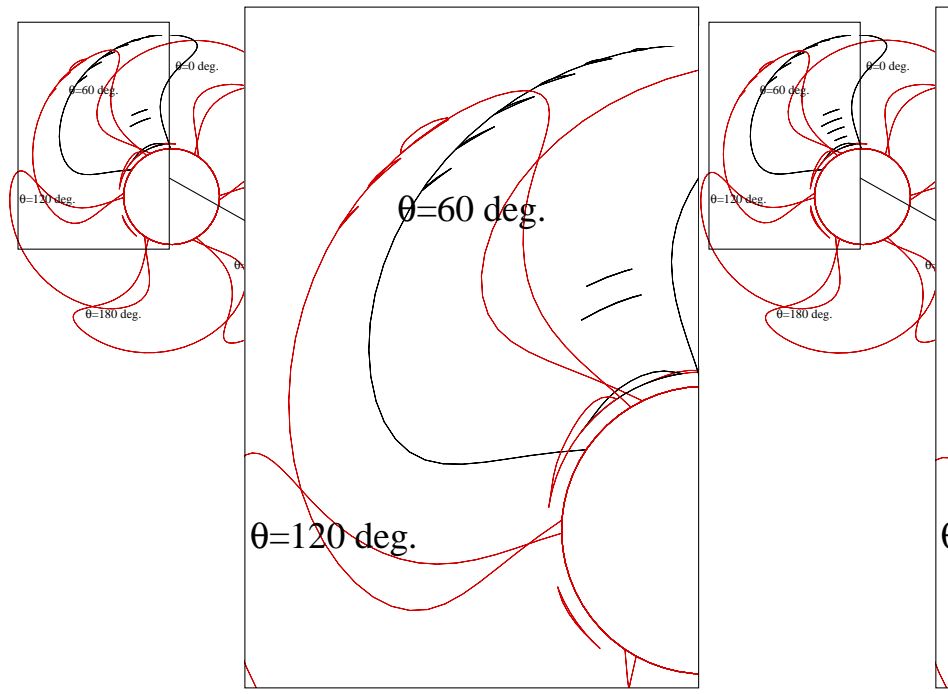

(c) Opt-5-8

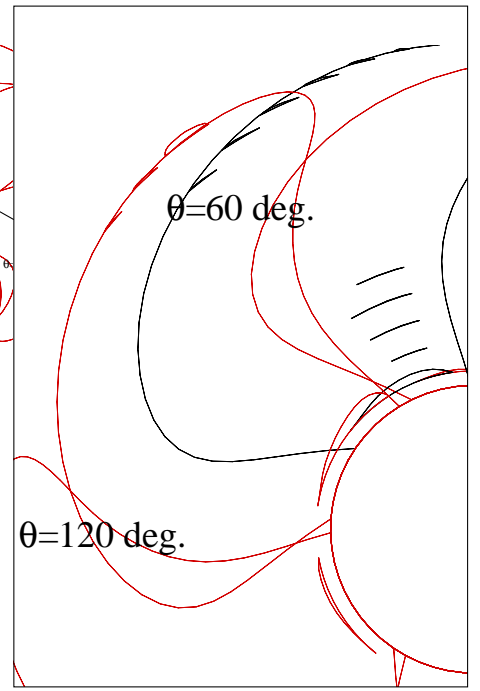

(d) Opt-5-9

Figure 21. Cavitation performance of propeller case Opt-5. (a) Opt-5-6; (b) Opt-5-13; (c) Opt-5-8; (d) Opt-5-9. 
The cavitation behaviour improves significantly even without amplification solely by enabling the cavitation centroid constraint (CCent). Opt-5-8 utilises this constraint and yields in general a CCent median of the baseline level, but suffers significant violation of $K_{T}$ with $88.1 \%$ of the variants. The improved mid-chord cavitation is shown in Figure 21c. This variant is selected from the Pareto front, satisfies the $K_{T}$ constraints and yields an improvement in both objectives ( $\eta=1.5 \%, p=-2.7 \%$ ).

The only optimisation case that distinctly reduces the violation of $K_{T}$ is Opt-5-9, which enables the amplification of the $K_{T}$ constraint. The number of variants that violates $K_{T}$ exclusively (31.8\%) and $K_{T}$ in combination with other constraints $(46.4 \%)$ is reduced to in total $78.2 \%$.

Optimisations in Group 3 aim to improve the cavitation behaviour by reducing the root cavitation and removing the entire mid-chord sheet cavitation. The baseline design features no mid-chord cavitation, but the increase of efficiency eases the inception speed and mid-chord cavitation is present for all results in Group 1 and 2. To still improve or maintain the objectives, we introduce parameters according to Table 12. Cavitation constraints are set to a threshold of a 5\% smaller maximal cavitation volume and cavitation length and a positive centroid harm-factor, which corresponds to an improvement of $-133 \%$.

Table 12. Parameter setting for support optimisation Opt-5.

\begin{tabular}{lc}
\hline Parameter Curve & Location $[r / R]$ \\
\hline Chord & 0.6 \\
Skew & 1.0 \\
Pitch & $0.4 \& 0.7$ \\
Camber & $0.4 \& 0.7$ \\
Rake & $0.7 \& 1.0$ \\
\hline
\end{tabular}

In Opt-5-11, the demand for a extreme centroid harm-factor reduction (CCent constraint) yields significant reduction of pressure pulses (median up to $-11 \%$, Figure A6c) and consequently no efficiency improvement. The cavitation volume and the centroid are, however, reduced by $80 \%$ and $150 \%$ respectively, while the $K_{T}$ is sacrificed and Opt-5-11 finalises the optimisation with a median $K_{T}$ of $-15 \%$ (Figure $22 b$ ).

Consequently, for Opt-5-14 and Opt-5-15 the high demand for the centroid harm-factor is reduced to a moderate enhancement of $33 \%$. This results in a better performance regarding efficiency and $K_{T}$, Figure A6c and Figure 22b. The geometry development is still the same, except for an opposite rake development for both rake modifications (Figure 22a RAKE1).

In Group 3, all Pareto solutions are violating the $K_{T}$ constraint. We select therefore designs that improve the objectives and predominantly satisfy the required $K_{T}$. Consequently, the gain in objectives is virtually not present (Table 13). The performance in Table 13 is given relative to the benchmark design. Although the cavitation volume is still larger than the benchmark, there is an improvement compared to the designs in Group 2. We compare the cavitation prediction of the benchmark design (Figure 23a) and the selected final designs in Figure 23 which provides quantitatively that the root cavitation is reduced in case of Opt-5-14 to the same level as the manual design and that the mid-chord cavitation vanishes. In terms of performance, the optimal design (Opt-5-15) is comparable with the benchmark, yet with $1.65 \%$ higher pressure pulses. The blade stress is reduced by $27.97 \%$ compared to the benchmark. The main conclusions regarding Opt-5 are summarised in Table 14. 


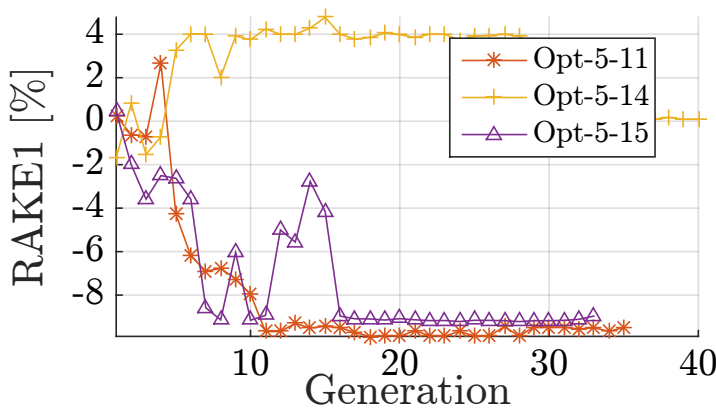

(a)

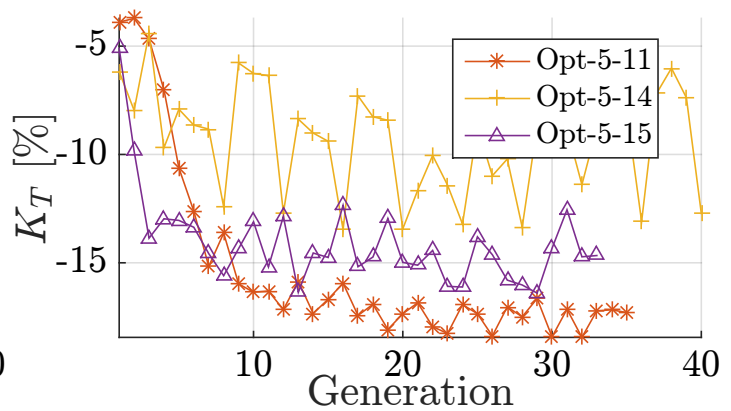

(b)

Figure 22. Group 3. (a) Rake development in Group 3 of Opt-5; (b) $K_{T}$ development in Group 3 of Opt-5.

Table 13. Performance of optimal solutions, relative to manual design performance.

\begin{tabular}{ccccccc}
\hline Optimisation & Variant & $\boldsymbol{\eta}[\%]$ & $\boldsymbol{p}[\%]$ & CavVol [\%] & FEM [\%] & $\boldsymbol{K}_{\boldsymbol{T}}[\%]$ \\
\hline Opt-5-11 & 47 & 0.27 & 4.55 & 30.961 & -19.35 & -0.27 \\
Opt-5-14 & 940 & 0.08 & 1.49 & 6.52 & -26.66 & 1.33 \\
Opt-5-15 & 287 & 0.38 & 1.65 & -3.09 & -27.97 & 0.09 \\
\hline
\end{tabular}

Table 14. Potential improvement and convergence observations of Opt-5.

\begin{tabular}{ll}
\hline Group & Observation \\
\hline & - Gain through optimisation is in case of Opt-5 substantial compared to the baseline \\
& $(\eta \geq 2 \%$ and $p \leq-8 \%$, Figure A6). \\
I & - Opt-5-4 reaches the top level efficiency improvement (median $\geq 2 \%)$. \\
& - Opt-5-1 yields a better trade-off between both objectives. \\
& Fast convergence in the parameters EAR, Skew tip, Rake tip and unload factor root; \\
& - The reduction of decision variables does not yield a positive effect on the number of \\
II & variants needed to convergence. \\
& - Less improvement potential due to strict constraints on the mid-chord cavitation \\
III & together with local modifications tailored for the cavitation improvement. \\
& - Underestimation of $p$ by meta-model of Opt-5-14.
\end{tabular}




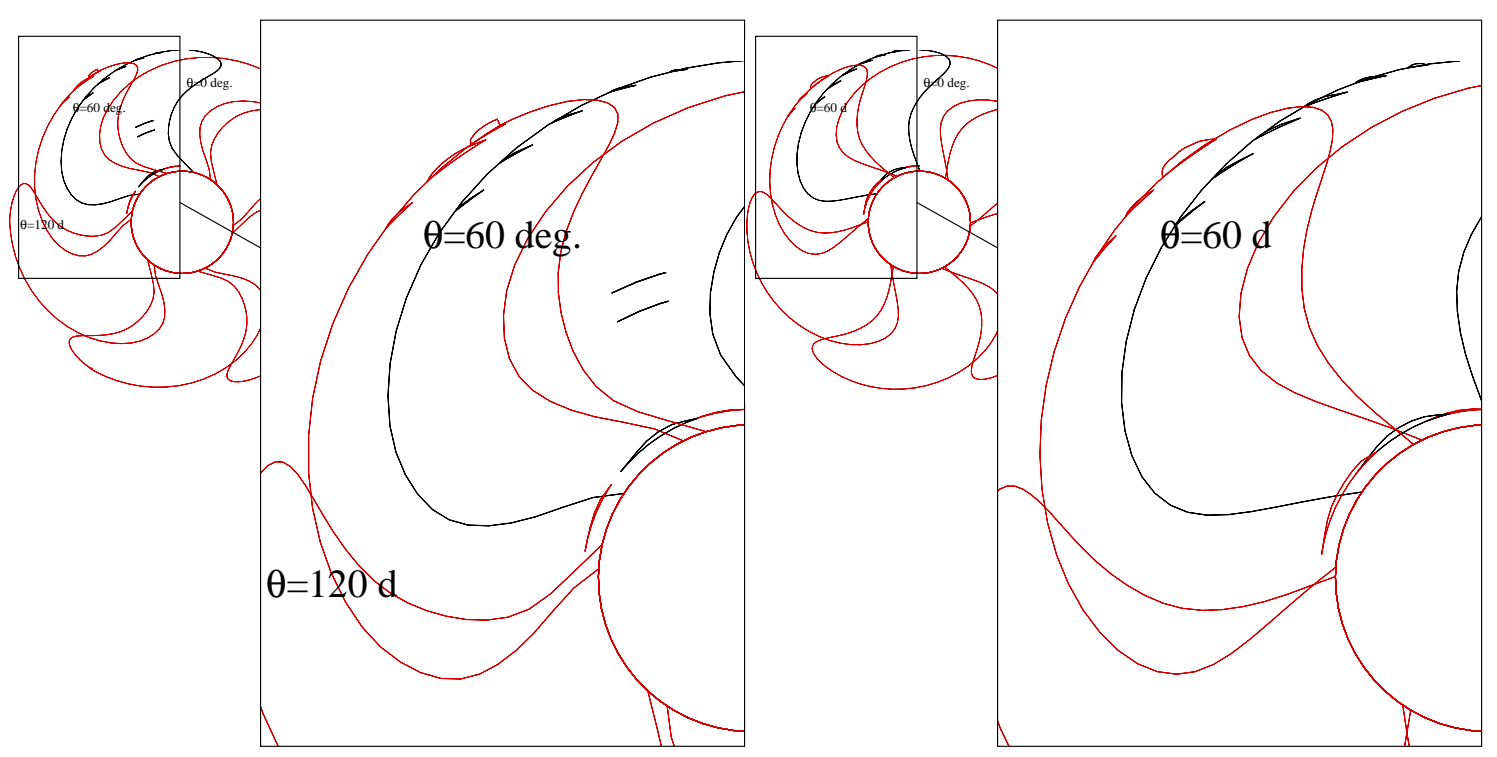

(a) Opt-5 benchmark

(b) Opt-5-11

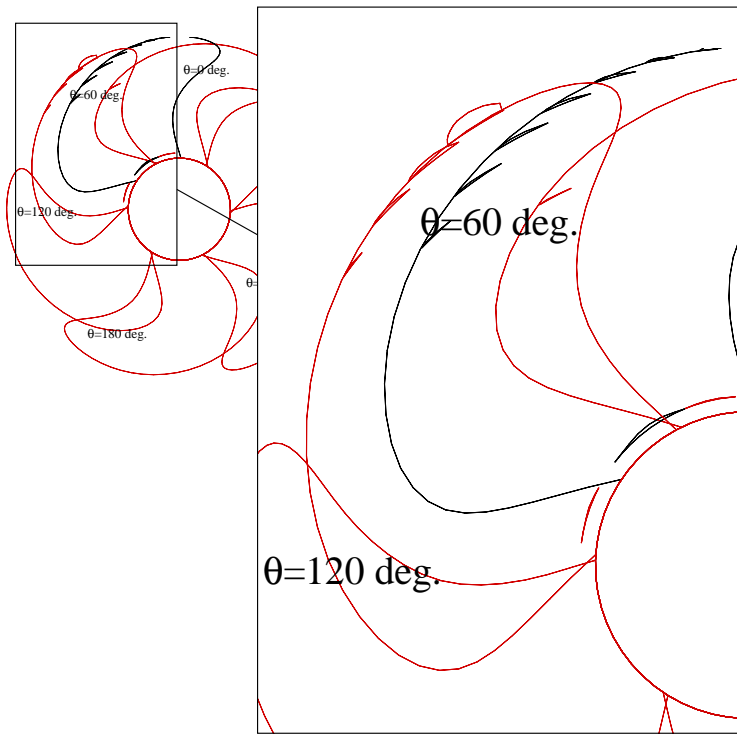

(c) Opt-5-14

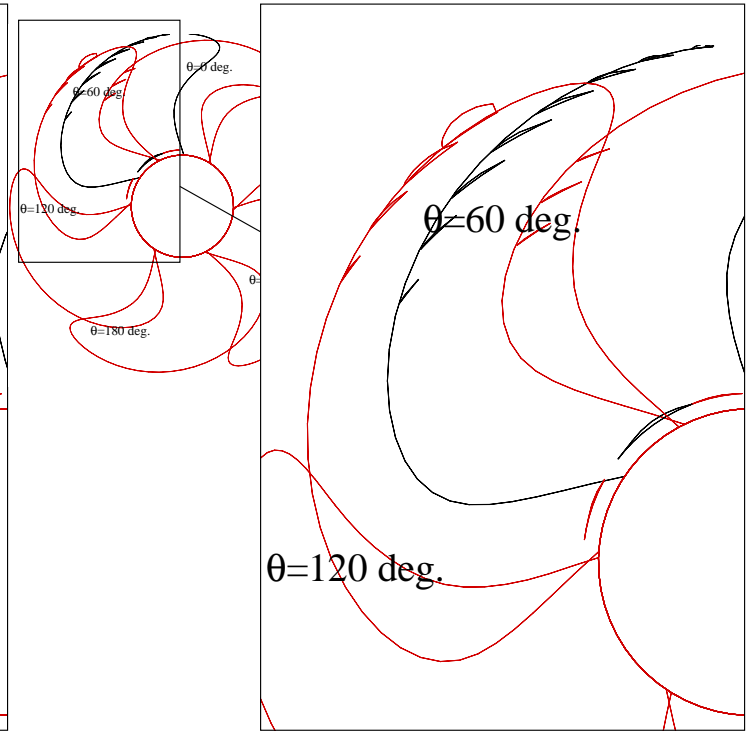

(d) Opt-5-15

Figure 23. Cavitation performance of propeller case Opt-5 in comparison with the manual benchmark design.

(a) Opt-5 benchmark; (b) Opt-5-11; (c) Opt-5-14; (d) Opt-5-15.

\subsubsection{Opt-6}

The convex closure line of the sheet cavitation can be predicted by MPUF-3A (Figure 24a) sufficiently well. Model tests and simulations with a boundary elements potential flow code confirm an even more pronounced convex closure line and the gap in radial directions of a cavitation free section around $r / R=0.4$. This case is therefore a favoured example to test the introduced cavity closure line constraints (Closure) and we are predominantly interested in the effect of that cavity shape constraint. Optimisation is hence only conducted within Group 3, aiming for a maintained efficiency and a sheet cavity that reduces the convexity of the cavity closure line and thereby reduce the risk for re-entrant jets and break-off. The harm-factor is intended to be improved by $-60 \%$ by applying in total six parameter changes according to Table 15 . The potential increase in efficiency is evident from 
Figure $24 \mathrm{c}$, which shows a continuous rise until it converges to $0.5 \%$ increase at generations 15 to 20 . The high threshold for the closure line harm-factor is, however, not achieved.

Table 15. Parameter setting for support optimisation Opt-5.

\begin{tabular}{lc}
\hline Parameter Curve & Location $[r / R]$ \\
\hline Chord & $0.6 \& 0.9$ \\
TMAX & 0.6 \\
Pitch & $0.6 \& 0.9$ \\
Camber & 0.6 \\
\hline
\end{tabular}

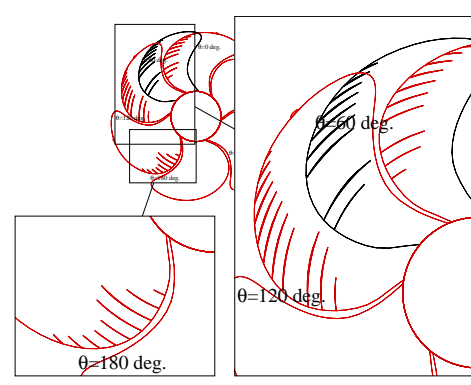

(a) Opt-6 bechmark

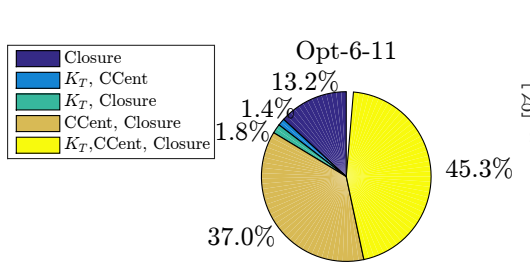

(b) Constraint violation

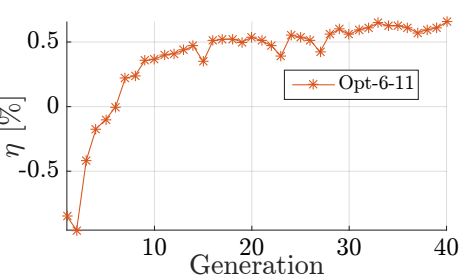

(c) Objective

Figure 24. Optimisation Opt-6, baseline cavitation performance (a); classification of individual constraints violation (b); and median performance of single-objective $\eta(\mathbf{c})$.

The parameter development is similarly blunt as the convergence of efficiency and most constraints indicate. Within about 20 generations all parameters gravitate towards a final value, which is in general a reduction in both chord length parameters of about $4 \%$, a mild reduction in camber, a significant reduction of thickness by $8 \%$ and in the pitch at the tip. All together, the changes indicate a dominance of the objective as driving force of the optimisation. Merely the thickness and camber reduction can be accounted to reduce the low pressure on the suction side and thereby the cavitation. A modification of camber and thickness distribution is preferable to locally change such cavitation.

Figure $24 \mathrm{~b}$ indicates that the closure line constraint is violated in $97.3 \%$ of the variants. The specified centroid target value is missed in $83.7 \%$ of the solutions. A detailed analysis of the constraints in Figure 25 reveals that mainly the cavity centroid (CCent) is improved. The maximal cavity volume (not active) still increases, compared with the benchmark design. The same holds for the cavity closure line constraint which increases up to $10 \%$.

However, the optimisation yields variants with improved cavitation behaviour, e.g., Opt-6-11-104 (Figure 26a) reduces the cavitation and more importantly changes the cavitation closure line such that the convexity vanishes with constant efficiency $(\eta=+0.007 \%)$ relative to manual benchmark design. The maximal volume reduces with 30\%. The variants Opt-6-11-334 and Opt-6-11-339 (Figure 26b,c) provide a different interpretation of the optimisation set-up, such that the cavitation satisfies the constraints in vicinity of the blade with the maximal volume and increases the efficiency, yet deteriorates the cavitation outside the range of considered blade positions. From these two variants, Opt-6-11-334 distinguishes itself by the best performance with regard to cavity centroid harm-factor, with an improvement of about 20\%. The variant Opt-6-11-532, with the best closure line constraint, provides long cavitating sections which reach the trailing edge of the blade (Figure 26d). This behaviour explains the low harm-factor for the closure line constraint since it deviates significantly from the given convex closure line and discloses thereby the weakness of that constraint. 


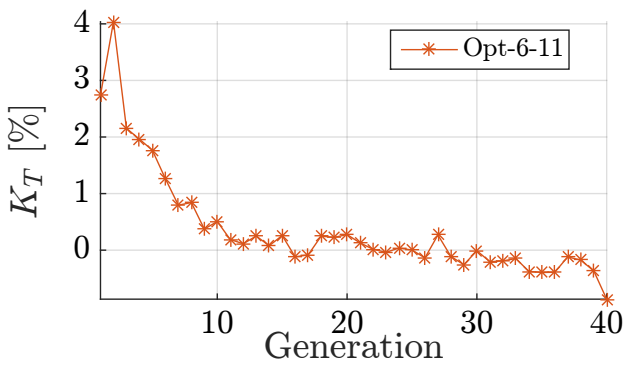

(a)

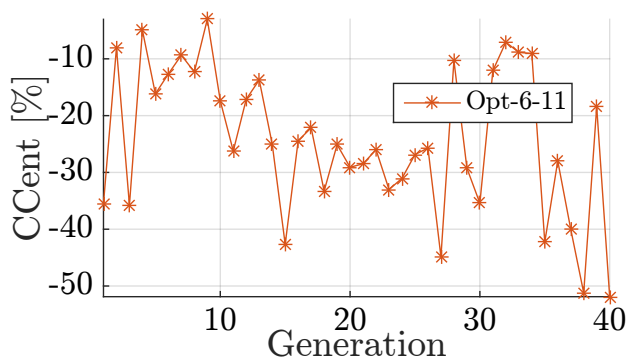

(c)

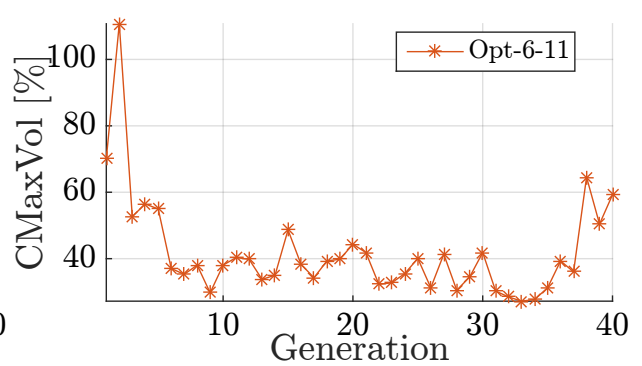

(b)

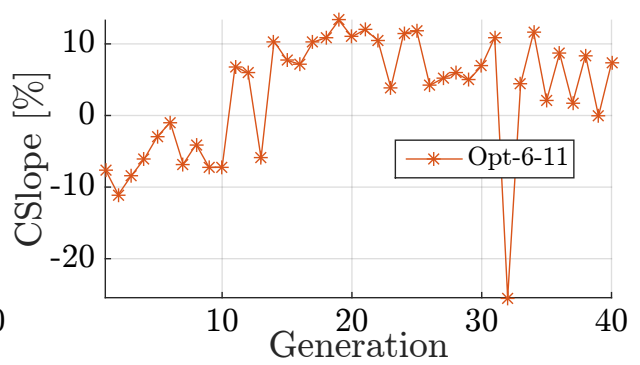

(d)

Figure 25. Development of constraints median throughout the optimisation. (a) $K_{T}$; (b) Cavity Volume; (c) Cavity Centroid; (d) Cavity Closure.

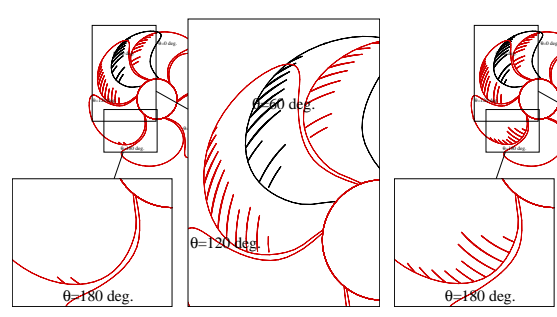

(a)

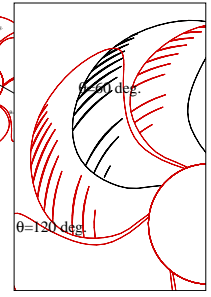

(b)

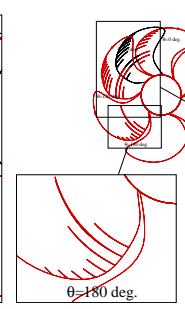

(c)

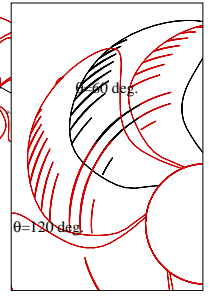

c)
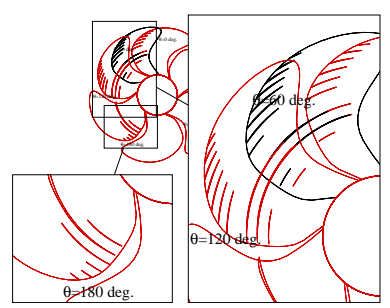

(d)

Figure 26. Cavitation performance of propeller case Opt-6. (a) Opt-6-11-104; (b) Opt-6-11-334; (c) Opt-6-11-339; (d) Opt-6-11-532.

\subsubsection{Opt-7}

In all optimisations, variants are created along a suboptimal Pareto front which is evident from the convergence of the optimisations in Figure 27 where the colour scale indicates the progress through the generation number. The optimisations find Pareto optimal solutions similarly located within the first generations, yet all converge to different location when the algorithms progresses. Opt-7-1, for instance converges to a broad band parallel to the Pareto front while Opt-7-2 gravitates to a region of $(-1 \% \eta,-20 \% p)$ and Opt-7-6 to $(0.6 \% \eta, 5 \% p)$ which is on the opposite end of the Pareto front. Opt-7-4 splits and converges to both ends of the Pareto front. This behaviour results, however, in a difference between Pareto optimal solutions and solutions of the converged optimisation, which is particularly obvious from the blade stresses.

Pareto optimal solutions yield designs with short chord length and suffer a severe violation of blade stresses (Figure 28). The designs are similar and the blade stresses of the Pareto optimal solutions are alike the baseline design, which is the consequence of lack of exploration since all designs are created at the beginning of the optimisation. Selecting, on the other hand solutions from the final generations reveals a notably improvement in blade stresses by all optimisation cases, Figure 29, 
which are more similar to the benchmark design (Figure 29a). Yet Opt-7-2 provides a design which is similar to the benchmark design in terms of blade loads (increase of only $6 \%$ ) while the pressure pulse objective is reduced by $25 \%$ (Table 16). The gain through optimisation is in case of Opt-7 small since the parametrised standard geometry curves offer not sufficient variation of the blade geometry. The chord length of the optimised solutions is, e.g., much shorter at the root than the manually designed blade (benchmark). The main conclusions regarding Opt-7 are summarised in Table 17.

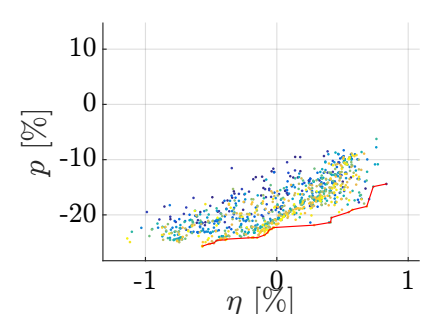

(a) Opt-7-1

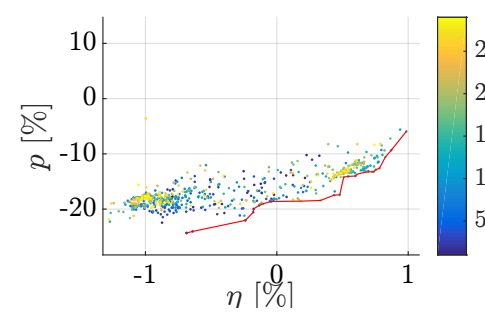

(c) Opt-7-4

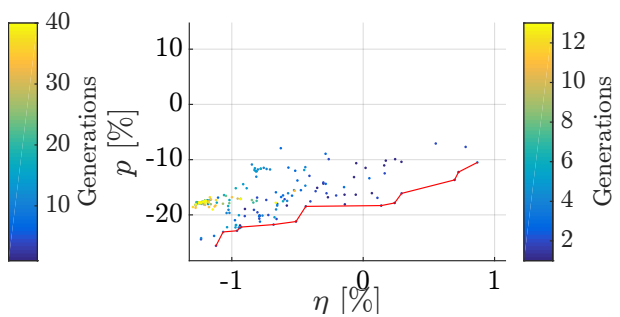

(b) Opt-7-2

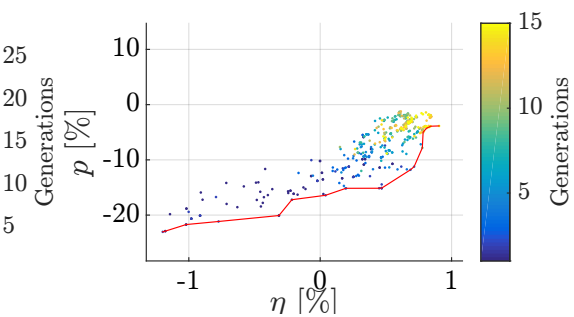

(d) Opt-7-6

Figure 27. Pareto fronts for Opt-5 for $\eta$ and pressure pulse objectives. (a) Opt-7-1; (b) Opt-7-2; (c) Opt-7-4; (d) Opt-7-6.

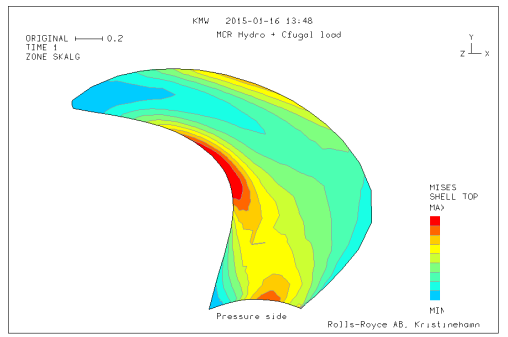

(a) Opt-7 baseline

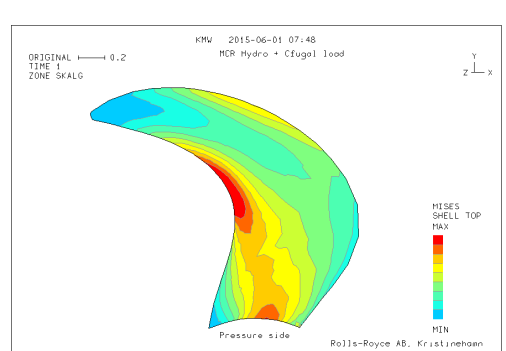

(c) Opt-7-2

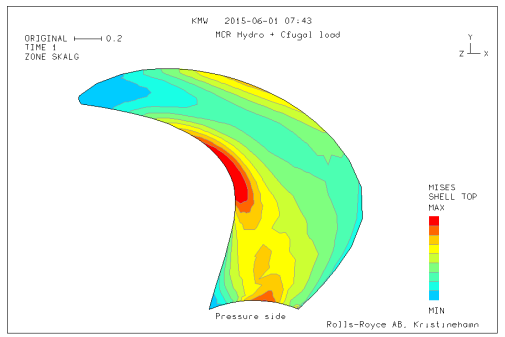

(b) Opt-7-1

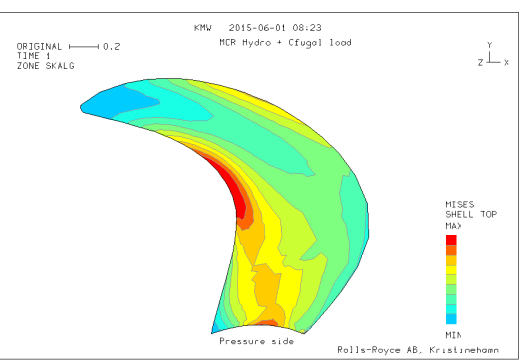

(d) Opt-7-4

Figure 28. FEM simulation of hydrodynamic and centrifugal loads of baseline design and Pareto optimal solutions in Group 1. (a) Opt-7 baseline; (b) Opt-7-1; (c) Opt-7-2; (d) Opt-7-4. 


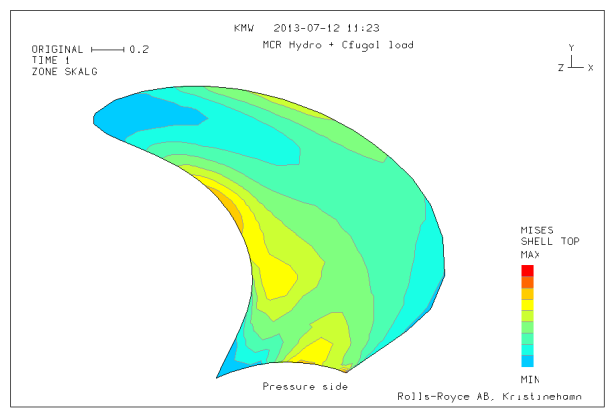

(a) Opt-7 benchmark

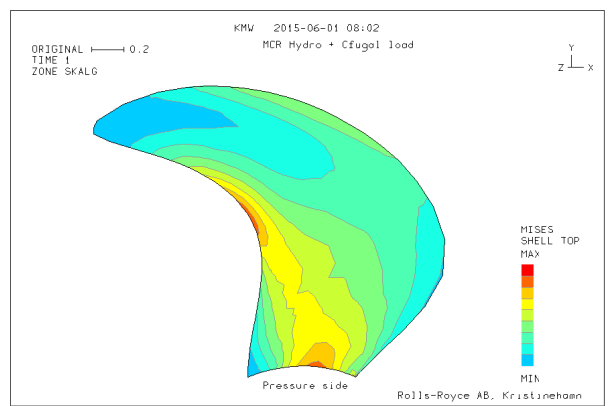

(c) Opt-7-2

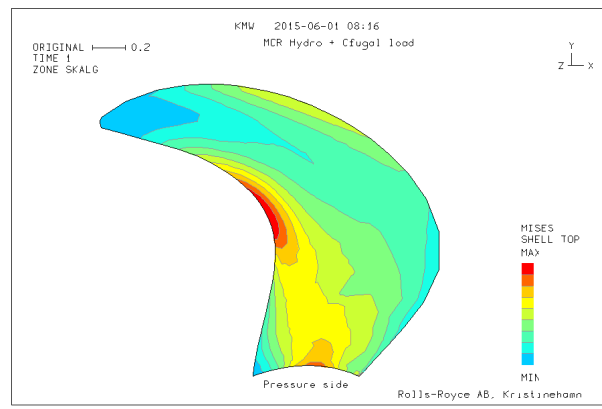

(b) Opt-7-1

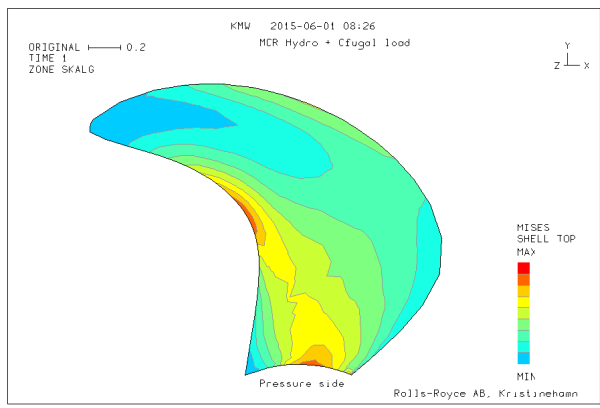

(d) Opt-7-4

Figure 29. FEM simulation of hydrodynamic and centrifugal loads of manual design and converged optimal solutions in Group 1. (a) Opt-7 benchmark; (b) Opt-7-1; (c) Opt-7-2; (d) Opt-7-4.

Table 16. Converged optimal solutions, relative to benchmark design.

\begin{tabular}{cccccc}
\hline Optimisation & Variant & $\boldsymbol{\eta}[\%]$ & $\boldsymbol{p}[\%]$ & FEM [\%] & $\boldsymbol{K}_{\boldsymbol{T}}[\%]$ \\
\hline Opt-7-1 & 808 & 0.17 & -30.09 & 35.40 & 2.45 \\
Opt-7-2 & 290 & -0.97 & -25.24 & 6.15 & 3.06 \\
Opt-7-4 & 677 & -0.77 & -25.45 & 8.78 & 3.15 \\
\hline
\end{tabular}

Table 17. Potential improvement and convergence observations of Opt-7.

\begin{tabular}{ll}
\hline Group & Observation \\
& - Potential gain in efficiency is the worst among the test cases (Figure A8). \\
& - Opt-7-1(NSGA-II) achieves a median value of a level similar to the baseline design. \\
& in Figure A8a). \\
- Already the baseline design yields a severe violation of approved maximal stress (Figure 28a). \\
- Opt-7-2 most distinct and rapid convergence. \\
- Opt-7-2 algorithm is the only that achieves the required reduction of stress (FEM in Figure 28c). \\
- Opt-7-4 algorithm balances the blade loading parameters and the EAR parameter. \\
- Opt-7-4 yields the second best stress reduction of around 20\%. \\
- Opt-7-3 and Opt-7-1 achieve in fact a reduction of blade stress, yet only a median \\
of about - 10\%. \\
- Reduction of input parameters, by omitting rake and skew line starting location at the hub, \\
results in better convergence. \\
- Opt-7-6 convergences strictly towards an improvement of $\eta$ and weight, disregarding the $p$ \\
objective (Figure 30b) and the blade stress constraint (Figure 30b).
\end{tabular}




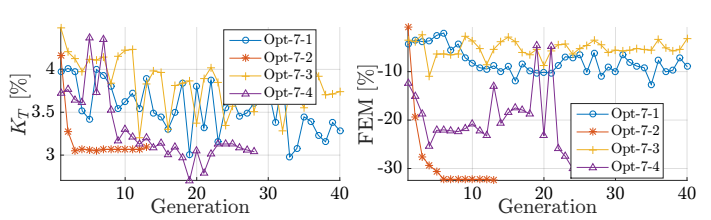

(a) Group 1

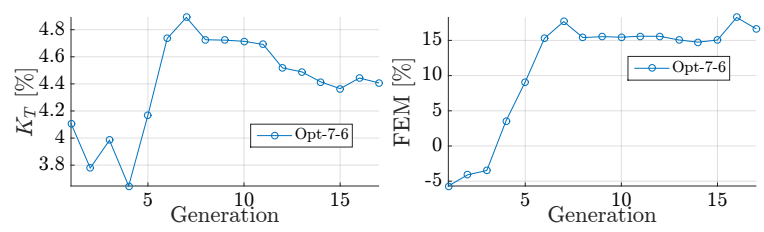

(b) Group 2

Figure 30. Development of objectives, given as median of relative improvements per generation. (a) Group 1; (b) Group 2.

\subsection{Summary of Results}

Frequently, the NSGA-II provided the best compromise between two objectives and converged in both objectives towards an approximation with fewest variation. Yet compliance with the constraints was insufficient. In many cases, either the CVM was violated or the NSGA-II was outperformed by the other algorithms regarding compliance of individual constraints, e.g., the required thrust or maximal blade stress. The blade stress reduction is achieved with larger improvement margin $(\approx-20 \%)$, which can thereby balance the CVM. However, the parameters that are likely to yield a stress reduction, i.e., chord length and blade thickness, are contradictory to the objectives. Thus, the optimisation should find a natural balance.

The proposed PSO and SANA NSGA-II algorithms perform similar in most of the cases regarding objectives and particular on adherence of constraints, which were, in contrast to the NSGA-II, more often fulfilled. The PSO is characterised, beyond the constraints compliance, also through a rapid convergence, but focuses on one of the objectives. The SANA NSGA-II (Opt-X-4) yields frequently to high fluctuations of EAR and unloading which balance each other: designs with a small EAR provide the advantage of less wetted surface (given that the blade radius is kept constant) and thereby less viscous drag which increases the efficiency. However, the smaller blade needs to compensate the generated thrust with a higher loading which again results in higher pressure pulse. The intense variation is related to the neighbourhood size in the SANA algorithm, which defines the variation by the standard deviation of a normal distribution. The AS NSGA-II performed, with the setting in Group 1 , not satisfactory. The estimations by the meta-model deviated widely from the calculated results which was obvious from the objectives and constraints. However, the meta-model adapted towards the end of the optimisation. In case of optimisations in Group 3, with localised modifications on the blade geometry, the AS NSGA-II performed significantly better and comparable with the NSGA-II, with the only exception of cruise ship propeller Opt-4.

In Group 2 the number of input parameters was reduced based on observations from Group 1 and supplementary with the results of a sensitivity analysis. Additionally, cavity shape constraints and amplification and constraints were introduced. The reduction of parameters, and thereby the design space, yielded, however, only improved convergence in one of the cases, independent whether additional shape constraints were applied. The cavity shape constraint on the cavity centroid harm-factor and the sheet thickness constraint performed well and managed to control the optimisation in the expected manner. Particularly during the support optimisation (Group 3) of the constraints category (Opt-3, Opt-5 and Opt-6), designs could be improved regarding cavitation utilising the shape constraints.

In comparison with the manually designed propellers, according to the established design procedure, the automated optimisation provide only in case of Opt- 4 insufficient performance. In that case, the parameters converged exceptionally rapidly towards the parameter limits. Opt- 4 baseline design deviated significantly from the benchmark design, which indicates that the parameter limits were too narrow to achieve the same performance. In the majority of the cases the optimisation yielded designs comparable with the manual work with advantages in constraints, e.g., lower blade loads or less cavitation or cavity shapes associated with higher margin to cavitation erosion, like removed mid-chord cavitation. The possible improvement in $\eta$ is only limited since the preliminary design 
philosophy yields designs that are already optimised to find the circulation distribution in a lifting-line and lifting-surface design method.

\section{Conclusions}

Manual propeller design is supreme to our current method. Designing a propeller incorporates many considerations regarding constraints and limitations, truly a multi-objective and multi-disciplinary optimisation task. An experienced designer considers regulations, limitations due to the type of propulsion systems (e.g., podded propulsion) and expected performance output with experience and makes reasonable decisions. The challenging factor in the manual design process is, however, the time which limits the possibilities to find the optimal design.

This paper presents optimisation algorithms and strategies applied to several propellers with different application purpose. In three cases the optimised design improved both, objectives and constraints, in three other cases the constraints are improved with maintained objectives, compared to the manual design. Only one case provided insufficient amendment due to deficient scope for development of the parameters. In all cases, optimal designs render, however, propeller designs that are comparable to the manually designed propeller blade geometries.

The optimisation handles multi-disciplinary evaluations and multiple constraints without user interaction. We recommend however a two-stage optimisation procedure with an assessment step in between to apply gathered knowledge and further exploit trends. The support optimisation, with local modification of the blade geometry, features particularly good performance to modify cavitation on the blade, together with the proposed cavity shape constraints. The AS NSGA-II is in this second optimisation phase comparable in performance with the NSGA-II and the SANA NSGA-II. The meta-model yields more accurate estimations due to reduced variation of the inputs. The AS NSGA-II is therefore beneficial in this stage as performance is better. The PSO or the SANA NSGA-II are advantageous in the primary optimisation due to superior constraint compliance. The aggregated constraint violation measure increases the flexibility and thereby the convergence of the optimisation. With amplification of a constraint, it is able to feature the designer's judgement of constraints but the CVM is greatly sensitive to the selection of constraints and their limits.

The purpose of this paper was to apply an automated optimisation procedure to a wide range of test propellers and evaluate the optimal results to determine propeller depending optimisation settings. The optimised propellers are, with respect to the applied settings, reasonably good; the designer may, however, consider design limitations that are beyond the applied evaluation methods. Yet, the generated designs guide the designer to find better solutions faster. To determine reliable guidelines for optimisation settings, further optimisations need to be assessed and categorised according to propeller design demands, e.g., a focus on cavitation, propeller efficiency or pressure pulse. In that context, the optimisation routine can be improved regarding stricter consideration of the $K_{T}$ constraint and in complexity, e.g., by consideration of several operation conditions or interchangeable settings of the objectives and constraints can be beneficial to achieve better and possibly novel designs faster.

Acknowledgments: This work has been carried out within the Rolls-Royce UTC in Computational Hydrodynamics at Chalmers, with the support from the Hydrodynamic Research Centre of Rolls-Royce AB.

Author Contributions: Florian Vesting (F.V.) is the main author of the paper; F.V. conceived the algorithm modifications and designed the optimisation strategies; F.V. carried out the optimisations and the assessment of the optimisation results; Rikard Johansson (R.J.) and Robert Gustafsson (R.G.) selected and prepared the test cases and contributed to the description of the test cases in the manuscript; Nicole Costa (N.C.) analysed the manual propeller design procedure; F.V. and Rickard E. Bensow (R.E.B.) carried out the discussion of results and analysed the optimisation strategies.

Conflicts of Interest: The authors declare no conflict of interest. 


\section{Abbreviations}

The following abbreviations are used in this manuscript:

$\begin{array}{ll}\text { AS NSGA-II } & \text { Adaptive Surrogate-assisted NSGA-II } \\ \text { CVM } & \text { Constraint Violation Measure } \\ \text { CPP } & \text { Controlable Pitch Propeller } \\ \text { EAR } & \text { Expanded Area Ratio } \\ \text { FPP } & \text { Fixed Pitch Propeller } \\ \text { PSO } & \text { Particle Swarm Optimisation } \\ \text { NSGA-II } & \text { Non-dominated Sorting Genetic Algorithm II } \\ \text { RRHRC } & \text { Rolls-Royce Hydrodynamic Research Centre } \\ \text { SANA NSGA-II } & \text { Surrogate-Assisted Neighbourhood Assessment NSGA-II }\end{array}$

\section{Appendix A. Additional Results Material}
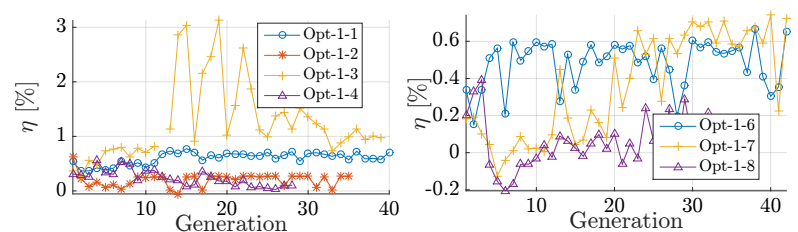

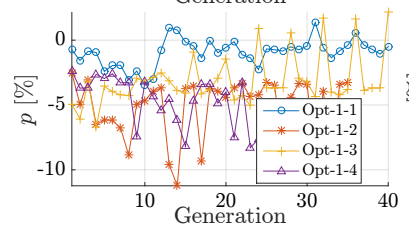

(a) Group 1

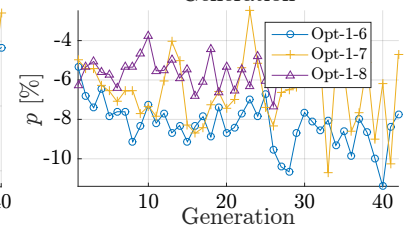

(b) Group 2

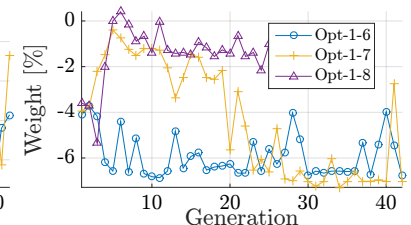

(c) Group 2

Figure A1. Development of objectives, given as median of relative improvements per generation. (a) Group 1; (b) Group 2; (c) Group 2.

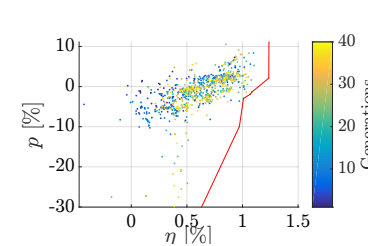

(a) Opt-1-1

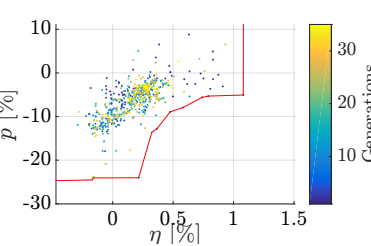

(b) Opt-1-2

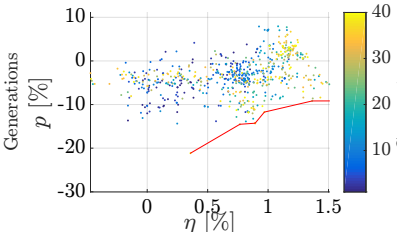

(c) Opt-1-3

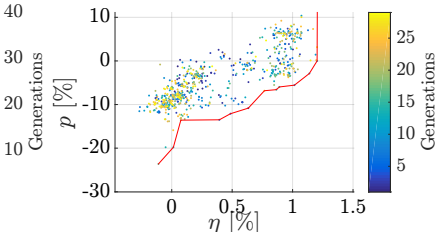

(d) Opt-1-4

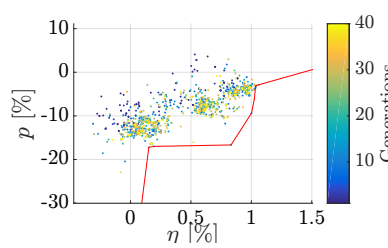

(e) Opt-1-6

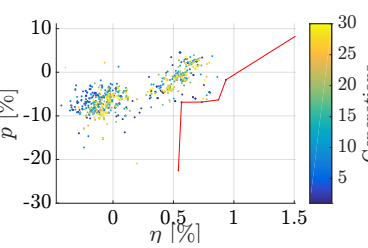

(f) Opt-1-7

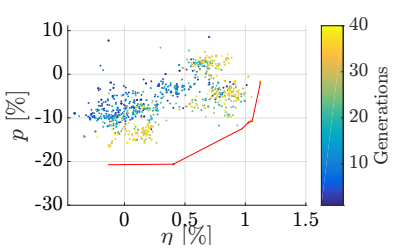

(g) Opt-1-8

Figure A2. Pareto fronts for Opt-1 for $\eta$ and pressure pulse objectives (Group 1: (a-d); Group 2: (f-g)). 

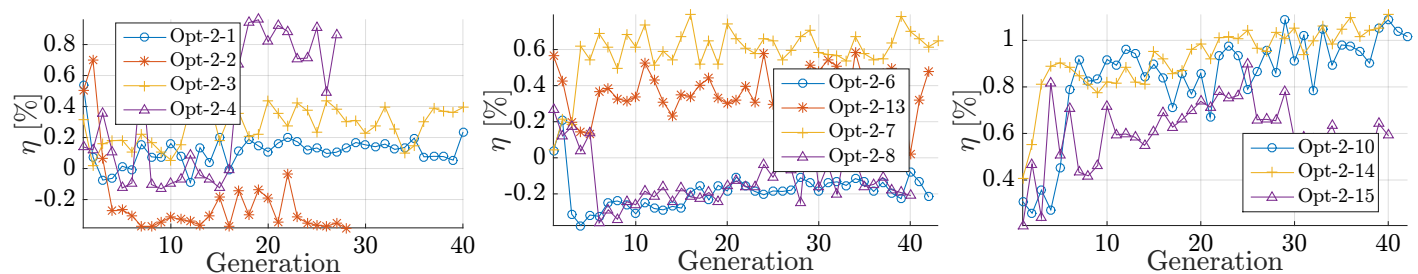

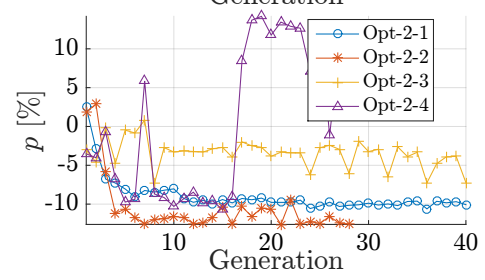

(a) Group 1

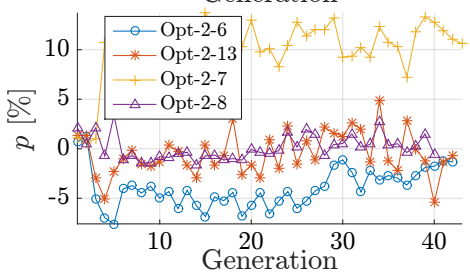

(b) Group 2

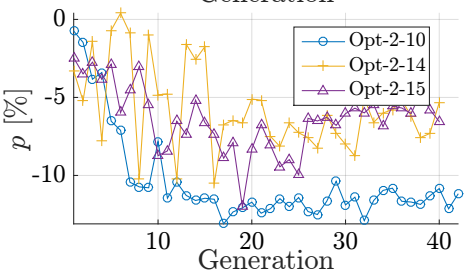

(c) Group 3

Figure A3. Development of objectives, given as median of relative improvements per generation. (a) Group 1; (b) Group 2; (c) Group 3.

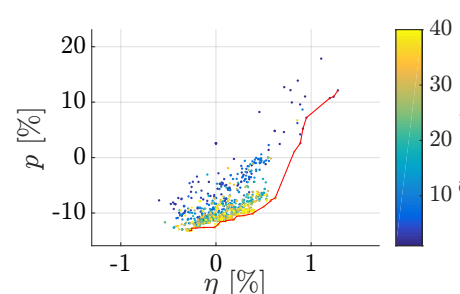

(a) Opt-2-1

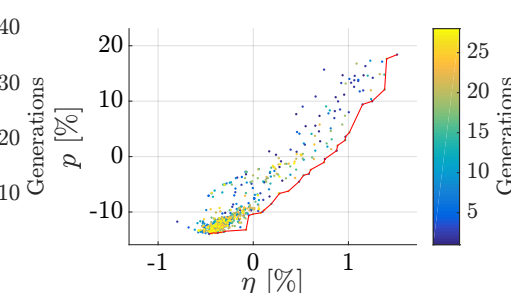

(b) Opt-2-2

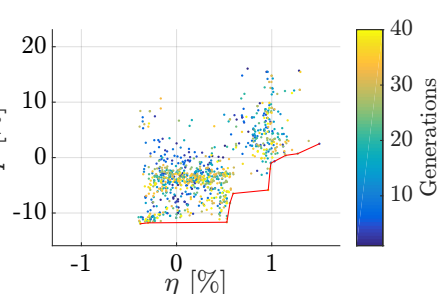

(c) Opt-2-3

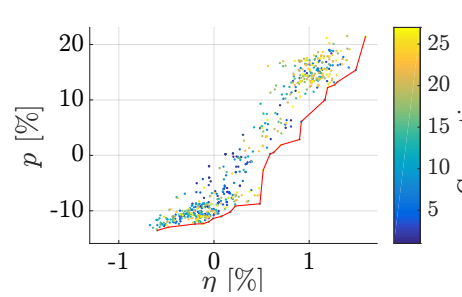

(d) Opt-2-4

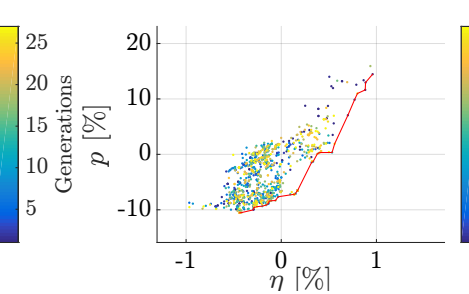

(e) Opt-2-6

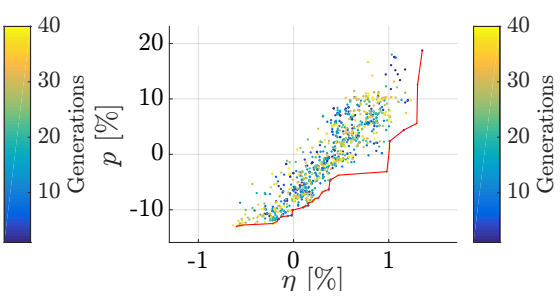

(f) Opt-2-13

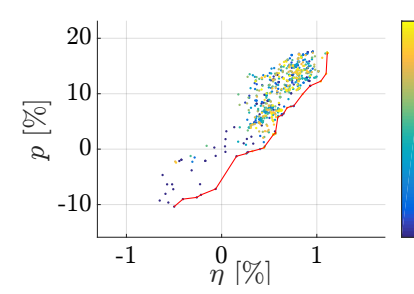

(g) Opt-2-7

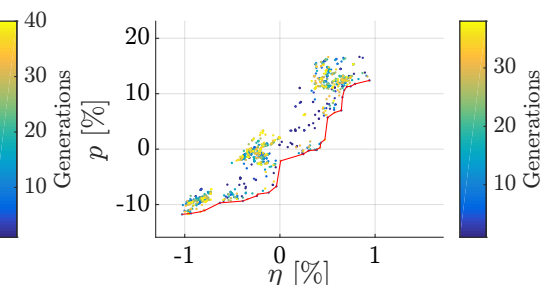

(h) Opt-2-8

Figure A4. Pareto fronts for Opt-2 for $\eta$ and pressure pulse objectives (Group 1: (a-d); Group 2: (f-h)). 

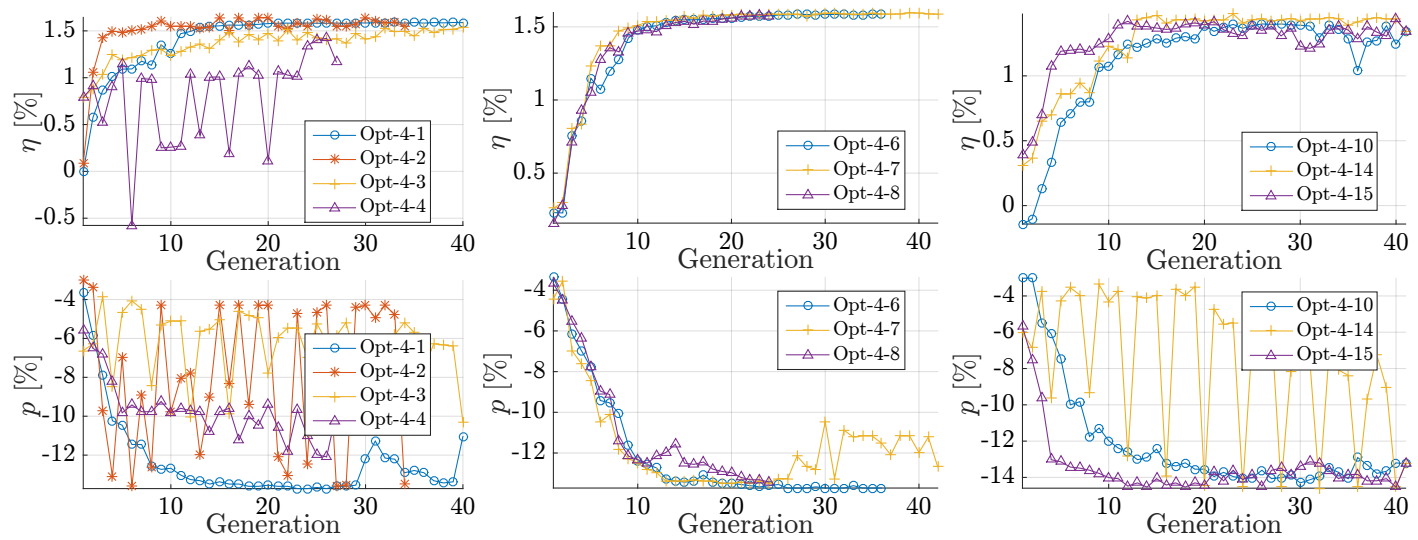

(a) Group 1

(b) Group 2

(c) Group 3

Figure A5. Development of objectives, given as median of relative improvements per generation. (a) Group 1; (b) Group 2; (c) Group 3.
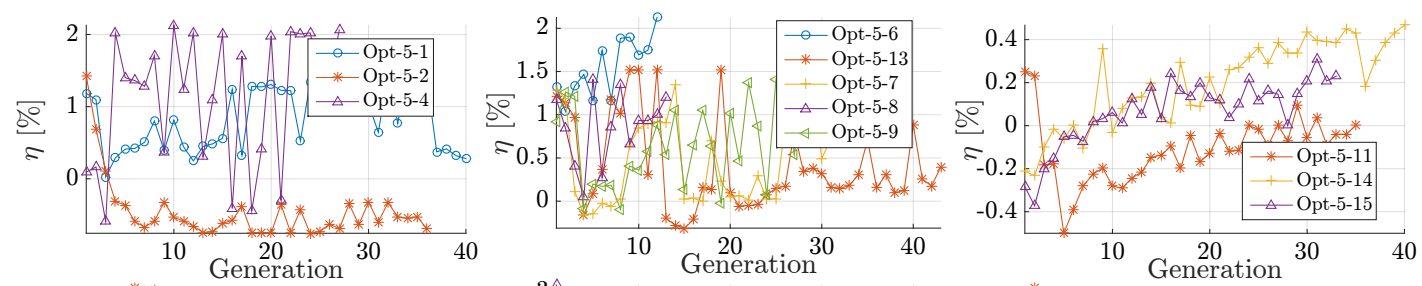

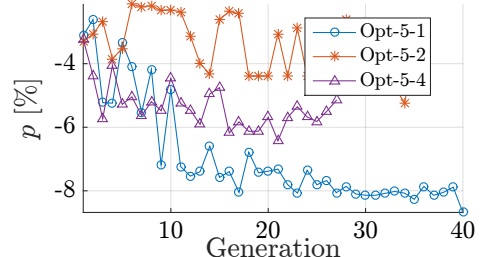

(a) Opt-5 Group 1

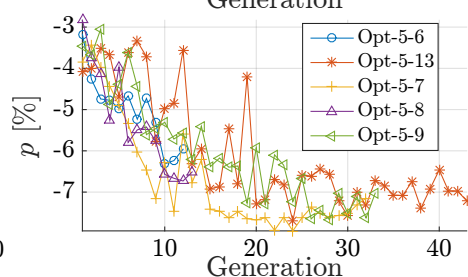

(b) Opt-5 Group 2

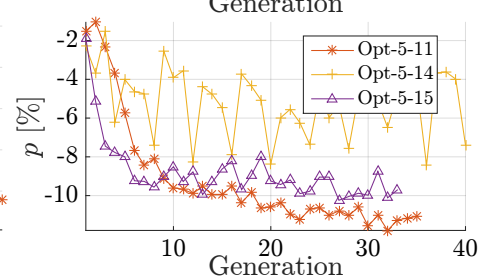

(c) Opt-5 Group 3

Figure A6. Analysis of the constraint violation in Group 2, with respect to the total number of solutions in each optimisation case. (a) Opt-5 Group 1; (b) Opt-5 Group 2; (c) Opt-5 Group 3.

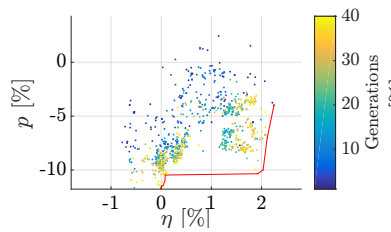

(a) Opt-5-1

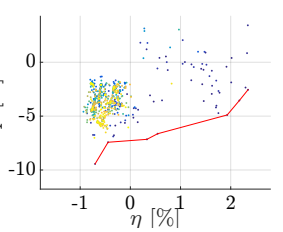

(b) Opt-5-2
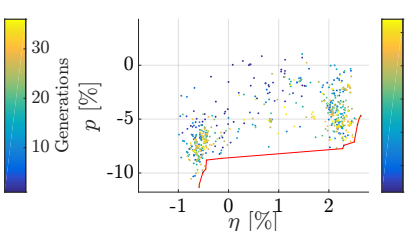

(c) Opt-5-4

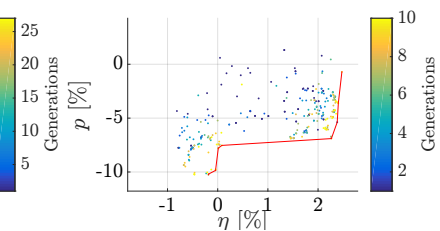

(d) Opt-5-6

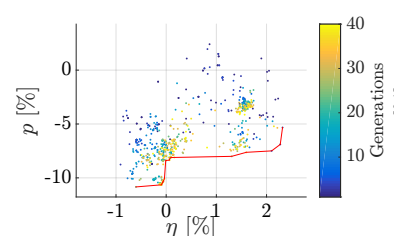

(e) Opt-5-13

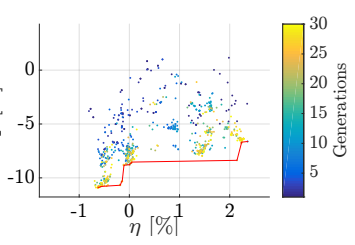

(f) Opt-5-7

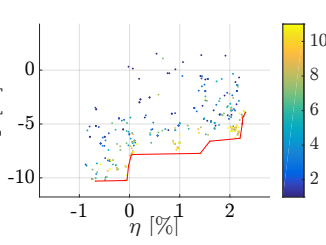

(g) Opt-5-8

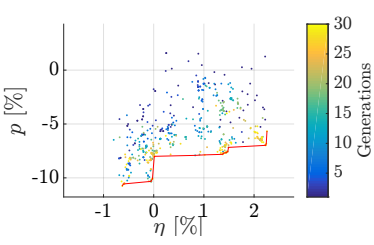

(h) Opt-5-9

Figure A7. Pareto fronts for Opt-5 for $\eta$ and pressure pulse objectives (Group 1: (a-d); Group 2: (f-h)). 

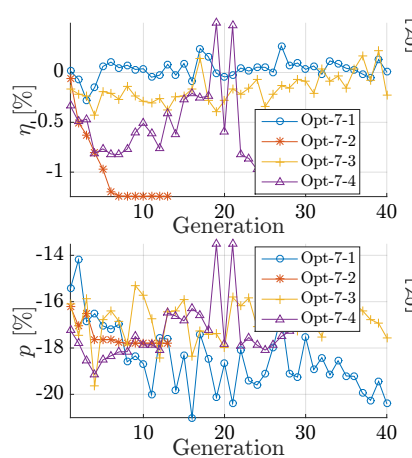

(a) Group 1
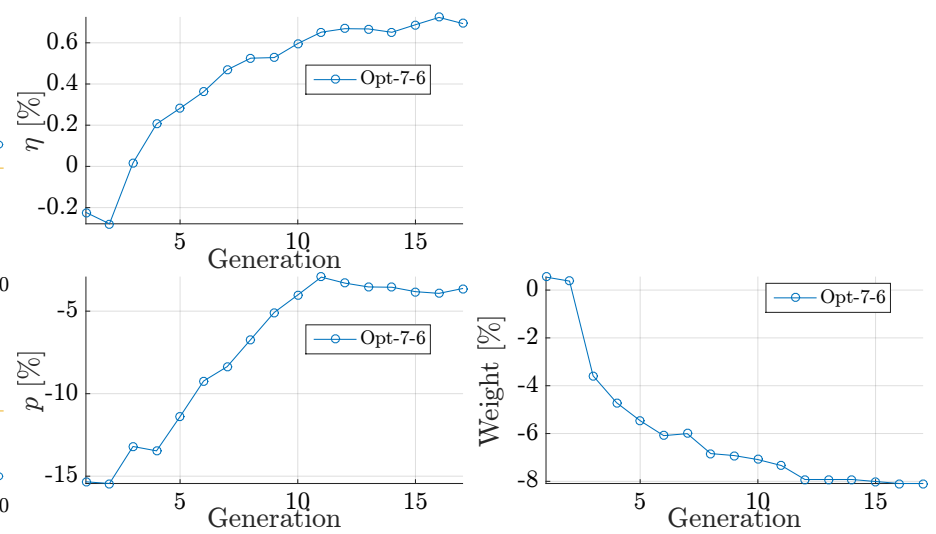

(b) Group 2

Figure A8. Development of objectives, given as median of relative improvements per generation. (a) Group 1; (b) Group 2.

\section{References}

1. Grunditz, G. Optimizing: Propeller and propulsion. Mar. Technol. 2015, 52, 48-55.

2. Mishima, S. Design of Cavitating Propeller Blades in Non-Uniform Flow by Numerical Optimization. Ph.D. Thesis, Massachusetts Institute of Technology, Cambridge, MA, USA, September 1996.

3. Griffin, P.E.; Kinnas, S.A. A Design Method for High-Speed Propulsor Blades. J. Fluids Eng. 1998, 120, 556-562.

4. Lee, C.S.; Choi, Y.D.; Ahn, B.K.; Shin, M.S.; Jang, H.G. Performance optimization of marine propellers. Int. J. Nav. Archit. Ocean Eng. 2010, 2, 211-216.

5. Bertetta, D.; Brizzolara, S.; Gaggero, S.; Viviani, M.; Savio, L. CPP propeller cavitation and noise optimization at different pitches with panel code and validation by cavitation tunnel measurements. Ocean Eng. 2012, 53, 177-195.

6. Foeth, E.J. Propeller optimization using an unsteady Boundary-Element Method. In Proceedings of the Fourth International Symposium on Marine Propulsors, Helsinki, Finland, 12-15 June 2017; Kinnas, S.A., Ed.; The University of Texas: Austin, TX, USA, 2015; Volume II, pp. 27-32.

7. Berger, S.; Druckenbrod, M.; Pergande, M.; Abdel-Maksoud, M. A Two-Stage Optimisation Method for Full-Scale Marine Propellers Working Behind a Ship. Ship Technol. Res. 2014, 61, 64-79.

8. Vesting, F.; Bensow, R. Propeller Optimisation Considering Sheet Cavitation and Hull Interaction. In Proceedings of the Second International Symposium on Marine Propulsors, Hamburg, Germany, 15-17 June 2011; Abdel-Maksoud, M., Ed.; Institute for Fluid Dynamics and Ship Theory: Hamburg, Germany, 2011; pp. $79-88$.

9. Han, K.J.; Bark, G.; Regnstrom, B. A Procedure for Optimizing Cavitating Propeller Blades in a Given Wake. Ship Technol. Res. 2006, 53, 39-52.

10. Kamarlouei, M.; Ghassemi, H.; Aslansefat, K.; Nematy, D. Multi-objective evolutionary optimization technique applied to propeller design. Acta Polytech. Hung. 2014, 11, 163-182.

11. Puisa, R.; Streckwall, H. Prudent constraint-handling technique for multiobjective propeller optimisation. Optim. Eng. 2011, 12, 657-680.

12. Deb, K.; Pratap, A.; Agarwal, S.; Meyarivan, T. A Fast Elitist Multi-Objective Genetic Algorithm: NSGA-II. IEEE Trans. Evolut. Comput. 2002, 6, 182-197.

13. Vesting, F.; Gustafsson, R.; Bensow, R.E. Development and application of optimisation algorithms for propeller design. Ship Technol. Res. 2016, 63, 50-69.

14. Lerbs, H.W. Moderately loaded propellers with a finite number of blades and an arbitrary distribution of circulation. In Proceedings of the Annual Meeting of The Society of Naval Architects and Marine Engineers, New York, NY, USA, 13-14 November 1952.

15. He, L.; Chang, S.; Kinnas, S. MPUF-3A Version 3.0; User Manual and Documentation; University of Texas: Austin, TX, USA, 2010. 
16. Kerwin, J.E. The Solution of Propeller Lifting Surfrace Problems by Vortex Lattice Methods; Technical Report; Massachusetts Institute of Technology: Cambridge, MA, USA, 1961.

17. Lee, C.S. Prediction of Steady and Unsteady Performance of Marine Propellers with or without Cavitation by Numerical Lifting-Surface Theory. Ph.D. Thesis, Massachusetts Institute of Technology, Cambridge, MA, USA, May 1979.

18. Kinnas, S.A. Non-Linear Corrections to the Linear Theory for the Prediction of the Cavitating Flow Around Hydrofoils. Ph.D. Thesis, Massachusetts Institute of Technology, Cambridge, MA, USA, May 1985.

19. Kerwin, J.; Kinnas, S.; Wilson, M. Experimental and analytical techniques for the study of unsteady propeller sheet cavitation. In Proceedings of the 16th Symposium on Naval Hydrodynamics, Berkeley, CA, USA, 13-16 July 1986; Webster, W.C., Ed.; Office of Naval Research, National Academy Press: Berkeley, CA, USA, 1986; pp. 387-414.

20. Sun, H.; Kinnas, S. HULLFPP, HULL Field Point Potential; User Manual and Documentation; University of Texas: Austin, TX, USA, 2007.

21. Sacks, J.; Welch, W.; Mitchell, T.; Wynn, H. Design and Analysis of Computer Experiments. Stat. Sci. 1989, 4, 409-435.

22. Lophaven, S.; Nielsen, H.; Søndergaard, J. DACE A Matlab Kriging Toolbox; Technical Report; Technical University of Denmark: Copenhagen, Denmark, 2002.

23. Kennedy, J.; Eberhart, R. Particle swarm optimization. In Proceedings of the IEEE International Conference on Neural Networks, Perth, Australia, 27 November-1 December 1995; Volume 4, pp. 1942-1948.

24. Saltelli, A.; Bolado, R. An alternative way to compute Fourier amplitude sensitivity test (FAST). Comput. Stat. Data Anal. 1998, 26, 445-460.

(C) 2016 by the authors; licensee MDPI, Basel, Switzerland. This article is an open access article distributed under the terms and conditions of the Creative Commons Attribution (CC-BY) license (http://creativecommons.org/licenses/by/4.0/). 